\title{
Fluorine Mass Balance and Suspect Screening in Marine Mammals from the Northern Hemisphere Supporting Information
}

Kyra M. Spaan ${ }^{1}$, Carmen van Noordenburg ${ }^{1}$, Merle M. Plassmann ${ }^{1}$, Lara Schultes ${ }^{1}$, Susan Shaw ${ }^{2}$, Michelle Berger ${ }^{2}$, Mads Peter Heide-Jørgensen ${ }^{3}$, Aqqalu Rosing-Asvid ${ }^{3}$, Sandra M. Granquist ${ }^{4,5}$, Rune Dietz ${ }^{6}$, Christian Sonne ${ }^{6}$, Frank Rigét ${ }^{6}$, Anna Roos ${ }^{3,7}$, Jonathan P. Benskin ${ }^{1 *}$

${ }^{1}$ Department of Environmental Science, Stockholm University, Svante Arrhenius Väg 8, 106 91, Stockholm, Sweden.

${ }^{2}$ Shaw Institute, P.O. Box 1652, Blue Hill, ME 04614

${ }^{3}$ Greenland Institute of Natural Resources, Nuuk, Greenland

${ }^{4}$ Marine and Freshwater Research Institute, Skúlagata 4, 101 Reykjavík, Iceland.

${ }^{5}$ The Icelandic Seal Center, Brekkugata 2, 530 Hvammstangi, Iceland

${ }^{6}$ Aarhus University, Department of Bioscience, Arctic Research Centre (ARC), Frederiksborgvej 399, PO Box 358, DK-4000 Roskilde, Denmark

${ }^{7}$ Department of Environmental Research and Monitoring, Swedish Museum of Natural History, Box 50007, 10405 Stockholm, Sweden

*Corresponding authors:

Kyra.Spaan@aces.su.se

Jon.Benskin@aces.su.se

Number of Pages: 38

Number of Figures: 13

Number of Tables: 10 


\section{Chemicals and Reagents}

Methanol (99.8\%, LiChrosolv®) and ammonium acetate (98\%) were purchased from Merck (Darmstadt, Germany). Acetonitrile ( $\geq 99.9 \%$, Chromasolv $\left.{ }^{\mathrm{TM}}\right)$ was obtained from Honeywell (France). Water was purified by a millipore water purification system and had a resistance $<18$ $\mathrm{M} \Omega / \mathrm{cm}$ (Milli-Q water). Fluoride standard (1000 mg/L) was obtained from Thermo Scientific. EnviCarb (Supelclean ${ }^{\mathrm{TM}}$ ) was obtained from Sigma Aldrich. Stainless steel beads $(4.8 \mathrm{~mm})$ were purchased from Next AdvanceC. Certified NIST serum (SRM 1957) was used for quality control. For CIC analysis, argon and oxygen gases were of purity grade 5.0 and the certified reference material (CRM) clay (BCR-461) was purchased from Sigma Aldrich.

\section{Sample preparation}

\section{Targeted analysis}

The extraction method was based on the method described by Powley et al. (2005) and was performed as follows. For each sample about $0.5 \mathrm{~g}$ of liver was thawed at room temperature and $50 \mu \mathrm{l}$ of internal standard (IS) solution $(20 \mathrm{pg} / \mu \mathrm{l})$ was added to each sample prior to extraction. Extraction was performed by adding $4 \mathrm{ml}$ acetonitrile $(\mathrm{ACN})$ together with 7-8 beads (stainless steel ø $4.8 \mathrm{~mm}$ ); thereafter the samples were homogenized in a bead blender (SPEX SamplePrep $1600 \mathrm{MiniG}\left({ }^{\circledR}\right)$ for $5 \mathrm{~min}$ at $1500 \mathrm{rpm}$. The samples were then centrifuged at $2000 \mathrm{rpm}$ for $5 \mathrm{~min}$ (Centrifuge 5810, Eppendorf, Hamburg) and the supernatant was transferred to a new $13 \mathrm{ml} \mathrm{PP-}$ tube. The precipitate was extracted one more time by adding another $4 \mathrm{ml} \mathrm{ACN}$, and vortexing, blending, and centrifuging again. The supernatant was added to the existing tube with the previous supernatant. The combined extracts were concentrated to $\sim 1 \mathrm{ml}$ under a stream of nitrogen in a water bath at $40{ }^{\circ} \mathrm{C}$ (TurboVap LV Evaporator, Biotage). The concentrated extracts were weighed and added to a $1.7 \mathrm{ml}$ Eppendorf tube containing $25 \mathrm{mg}$ EnviCarb and $50 \mu \mathrm{l}$ acetic acid. The tubes were vortexed and centrifuged for $10 \mathrm{~min}$ at $10000 \mathrm{rpm}$ (Galaxy 14D, Microcentrifuge, VWR). Then $500 \mu \mathrm{l}$ of the supernatant were transferred to another Eppendorf tube. To this $50 \mu$ recovery standard (RS) solution (20 pg/ $\mu 1{ }^{13} \mathrm{C}_{8}$-PFOA and ${ }^{13} \mathrm{C}_{8}$-PFOS) and $500 \mu \mathrm{NH}_{4} \mathrm{OAc}(4 \mathrm{mM}$ in water) were added and the extracts were stored at $-20{ }^{\circ} \mathrm{C}$ until analysis. On the day of analysis, the extract was adjusted to room temperature, vortex-mixed and transferred to an LC vial.

\section{Clean-up step test}


Two clean-up steps were evaluated for their potential to remove inorganic fluorine and recovery of target analytes: 1) a solid phase extraction (SPE)-based clean-up, and 2) an EnviCarb-based clean-up. The SPE extraction method was based on Miyake et al. ${ }^{2}$ and the EnviCarb extraction on Powley et al. ${ }^{1}$ Fish muscle samples were spiked with $250 \mathrm{ng}$ PFOS ( 162 ng F) and $500 \mathrm{ng} \mathrm{NaF.}$ Method blanks showed high concentrations for the SPE clean-up with high variation, while the method blanks for the EnviCarb clean-up step were rather low and consistent. The unspiked samples showed similar concentrations for both methods, however EnviCarb showed a bit higher deviation. PFOS and $\mathrm{NaF}$ recoveries were calculated according to the following formula:

$$
\text { Recovery }(\%)=\frac{\text { Measured spike (ng F) }- \text { Measured no spike (ng F) }}{\text { Spiked concentration (ng F) }} \times 100 \%
$$

PFOS recovery was high for both methods, i.e. $96 \%$ and $92 \%$ for SPE and EnviCarb, respectively. Both extraction methods aim to remove inorganic fluorine, such as $\mathrm{NaF}$, and get as low recovery as possible. NaF recovery was $12.5 \%$ and $-0.2 \%$ for SPE and EnviCarb, respectively. Only the EnviCarb method was able to remove the inorganic fluorine effectively. After this extraction method comparison, EnviCarb was found to be the best suitable to use for analysis of the real samples, since this approach resulted in lower method blanks and more efficient removal of inorganic fluorine and was therefore considered the most suitable clean-up method.

\section{Total and extractable organofluorine}

A similar extraction procedure was applied to the liver samples prior to analysis with the CIC. Since the CIC measures the total fluorine concentration, no internal standards were added, also no $\mathrm{NH}_{4} \mathrm{OAc}$ was added in the end. The final extracts $(\sim 1 \mathrm{ml})$ were split into two parts, in order to have a replicate of each sample. Also, since the sample boats have limited sample space, the final split extracts were concentrated to $\sim 200 \mu 1$ under a stream of nitrogen.

\section{Instrumental Analysis}

\section{Targeted analysis}

The system was operated in negative ion electrospray ionization (ESI-) mode. The source and desolvation temperatures were set at $150{ }^{\circ} \mathrm{C}$ and $350{ }^{\circ} \mathrm{C}$, respectively. The desolvation and cone gas flows (nitrogen) were set at $650 \mathrm{~L} / \mathrm{h}$ and $150 \mathrm{~L} / \mathrm{h}$, respectively. The capillary voltage was set at $1.0 \mathrm{kV}$. Qualification and quantification were carried out using MassLynx 4.1 (Waters). 
Quantification was performed using internal standards via a 9-point calibration curve ranging from 0.008 to $150 \mathrm{ng} / \mathrm{ml}$ (linear, 1/x weighting). Precursor and product ions are presented in Table S7. Analytes lacking an analogous labeled standard were quantified using the IS with the closest retention time and the data quality was defined as semi-quantitative (semiQ). Branched isomers were quantified using the calibration curve of the linear isomer.

\section{Total and extractable organofluorine analysis}

Measurements of total fluorine (TF) and extractable organofluorine (EOF) were carried out using a Thermo-Mitsubishi CIC using previously described methods. ${ }^{3,4}$ Briefly, extracts ( 200 $\mu 1$ for samples and $100 \mu \mathrm{l}$ for standards) were placed in a ceramic sample boat containing glass wool (for fluid dispersion), while neat liver material ( 100 mg) was weighed directly into the sample boat. The samples were combusted slowly in a horizontal furnace (HF-210, Mitsubishi) at $1100{ }^{\circ} \mathrm{C}$ under a flow of oxygen $(400 \mathrm{ml} / \mathrm{min})$, argon $(200 \mathrm{ml} / \mathrm{min})$, and argon mixed with water vapor $(100 \mathrm{ml} / \mathrm{min})$ for approximately 5 minutes. Combustion gases were absorbed in MilliQ water during the entire length of the combustion process using a gas absorber unit (GA-210, Mitsubishi). A $200 \mu \mathrm{l}$ aliquot of the absorption solution was subsequently injected onto an ion chromatograph (Dionex Integrion HPIC, Thermo Fisher Scientific) equipped with an anion exchange column

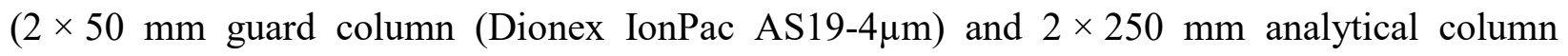
(Dionex IonPac AS19-4 $\mu \mathrm{m}$ ) operated at $30^{\circ} \mathrm{C}$. Chromatographic separation was achieved by running a gradient of aqueous hydroxide mobile phase ramping from $8 \mathrm{mM}$ to $100 \mathrm{mM}$ at a flow rate of $0.25 \mathrm{ml} / \mathrm{min}$ (Table S8), and fluoride was detected using a conductivity detector. Quantification was carried out using a standard calibration curve prepared at 0.05 to $100 \mu \mathrm{g} \mathrm{F} / \mathrm{ml}$. The calibration curve showed very good linearity with $\mathrm{R}^{2}>0.98$. The mean fluoride concentration in the method blanks was subtracted from the samples. The method detection limit (MDL) was defined as the mean concentration plus three times the standard deviation in the method blanks.

\section{Suspect screening}

Suspect screening was carried out using a Dionex Ultimate 3000 liquid chromatograph coupled to a Q Exactive HF Orbitrap (Thermo Scientific), based on a previously described method. ${ }^{5}$ The flow rate was held constant at $0.4 \mathrm{ml}$ per minute throughout the run. The mobile phases and eluent program used for non-target/suspect screening were the same as those used for target analysis (i.e. 
by UPLC-MS/MS). The instrument was run in negative ion, full scan $(200-1200 \mathrm{~m} / \mathrm{z})$ data dependent acquisition (DDA) MS/MS mode (50-1200 m/z). The resolution was set to 120000 (15 000 for MS/MS), the automatic gain control (AGC) was set to 3e6, and other instrumental parameters are presented in Table S11. Briefly, $\mathrm{CL}=5$ is assigned when only the exact mass is known. $\mathrm{CL}=4$ is used when the unknown analyte ion can be assigned an unambiguous formula, but no structural information is available. $\mathrm{CL}=3$ represents tentative candidates whose possible structure can be proposed but lack sufficient information to assign an exact structure. $C L=2 a$ represents probable structures by comparing to library spectra where spectrum-structure is unambiguous. $\mathrm{CL}=2 \mathrm{~b}$ can be assigned when no standard or literature information is available for confirmation and there is only diagnostic evidence. Finally, $\mathrm{CL}=1$ represents confirmed structures, that match a reference standard with MS, MS/MS and RT.

\section{Quality Control}

\section{Targeted analysis}

Limits of quantification (LOQs) were determined by the lowest calibration concentration that showed a well-shaped peak with intensity $>1 \mathrm{e} 3$ and signal-to-noise $(\mathrm{S} / \mathrm{N})>3$. For compounds that were not present in the calibration standard, but that were detected in the samples (PFPeDA, PFHpS, and branched isomers), the LOQ from the corresponding standard was used. For compounds where method blank contamination was observed (PFBS, PFOS, and FOSAA), the LOQ was determined as the average of the quantified concentrations in the method blanks plus ten times the standard deviation. The compound-specific LOQs are listed in Table S6.

Method accuracy and precision for most substances was very good, with percent recoveries ranging from $73-130 \%$ and standard deviations ranging from 3-30\% (Figure S2). The exceptions were for PFHxDA, PFOcDA, 4:2 FTSA, and 8:2 FTSA, which showed very high recoveries (278\%, 397\%, 212\%, and 227\%, respectively), while HFPO-DA, 3:3 FTCA, 5:3 FTCA, and 7:3 FTCA showed very low recoveries $(22 \%, 34 \%, 55 \%$, and $53 \%$, respectively). These deviating recoveries are likely due to matrix effects, which were not accounted for because of the absence of an exactly matching isotopically-labeled internal standard. Nevertheless, the targets with very high recoveries were included in the analysis, since their concentrations in the samples were so low $(<1 \mathrm{ng} / \mathrm{g}$, ww). The targets with low recoveries were also included in the analysis, albeit 
measured concentrations may be underreported. Finally, the method was externally validated by analyzing a standard reference material (SRM) sample of NIST serum 1957. Results are presented in Table S9 and were generally in good agreement with certified values and prior measurements of this material by other researchers.

\section{Total and extractable organofluorine}

All boats were baked out prior to sample combustion to minimize background contamination. Each run started and ended with a calibration curve and after every 8-10 samples, a blank and a midlevel calibration standard were analyzed for quality control. The removal efficiency of inorganic fluoride was tested by spiking a range of known concentrations of $\mathrm{NaF}(0.25,0.5,0.75,1$, and 2 $\mu \mathrm{g}$ ) into liver tissue followed by extraction (Figure S3). Furthermore, recovery of organic fluoride was determined by spiking PFOS $(0.08,0.13,0.25,0.5$, and $1 \mu \mathrm{g} / \mathrm{ml})$ to liver tissue and performing the extraction (Figure S4). CIC analysis of both the extracted liver residue and the EnviCarb used for clean-up showed that the inorganic fluoride remained in the extracted liver; in other words, it was not extracted during the initial acetonitrile extraction step. The obtained recovery for PFOS was used to correct the measured concentrations of EOF in real samples. In theory, since the recovery is different for each target analyte, the recovery should be determined for each individual compound. However, practically this would mean a large number of experiments and therefore only the recovery for the most abundant compound, PFOS, was assessed here. 


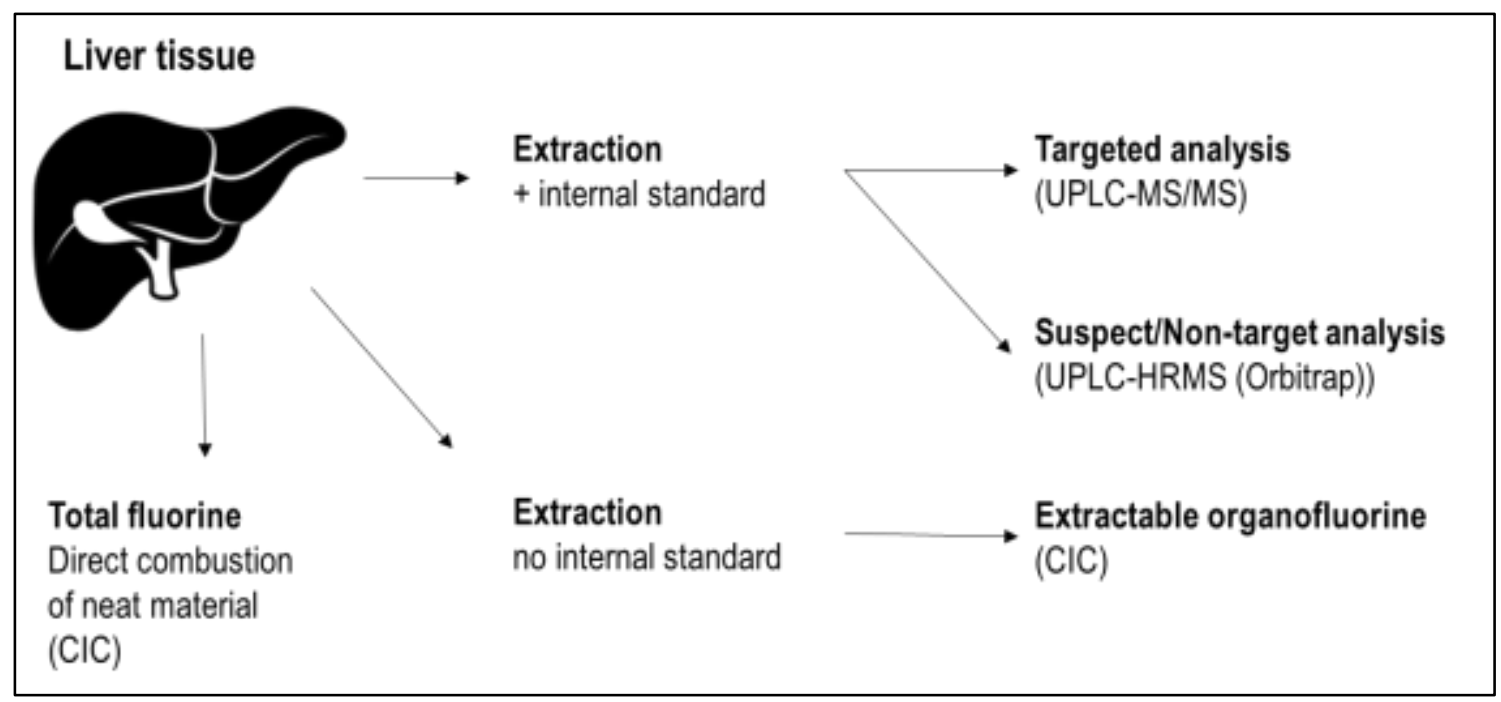

Figure S1. Diagram of the experimental design. 

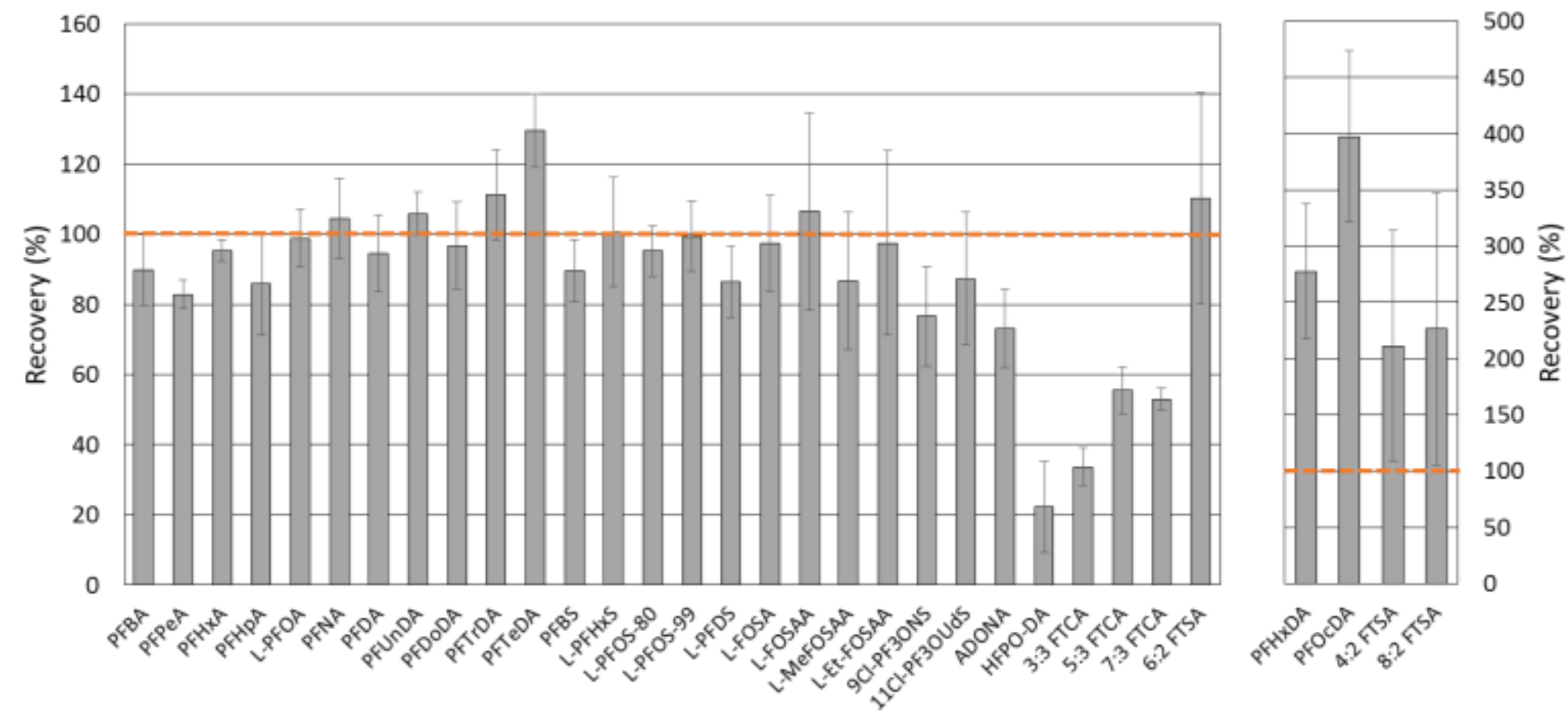

Figure S2. Recovery \pm standard deviation (\%) of native compounds spiked in seal samples $(n=4)$. Right panel shows severe overrecovery of four targets, attributable to matrix-induced ionization enhancement. 


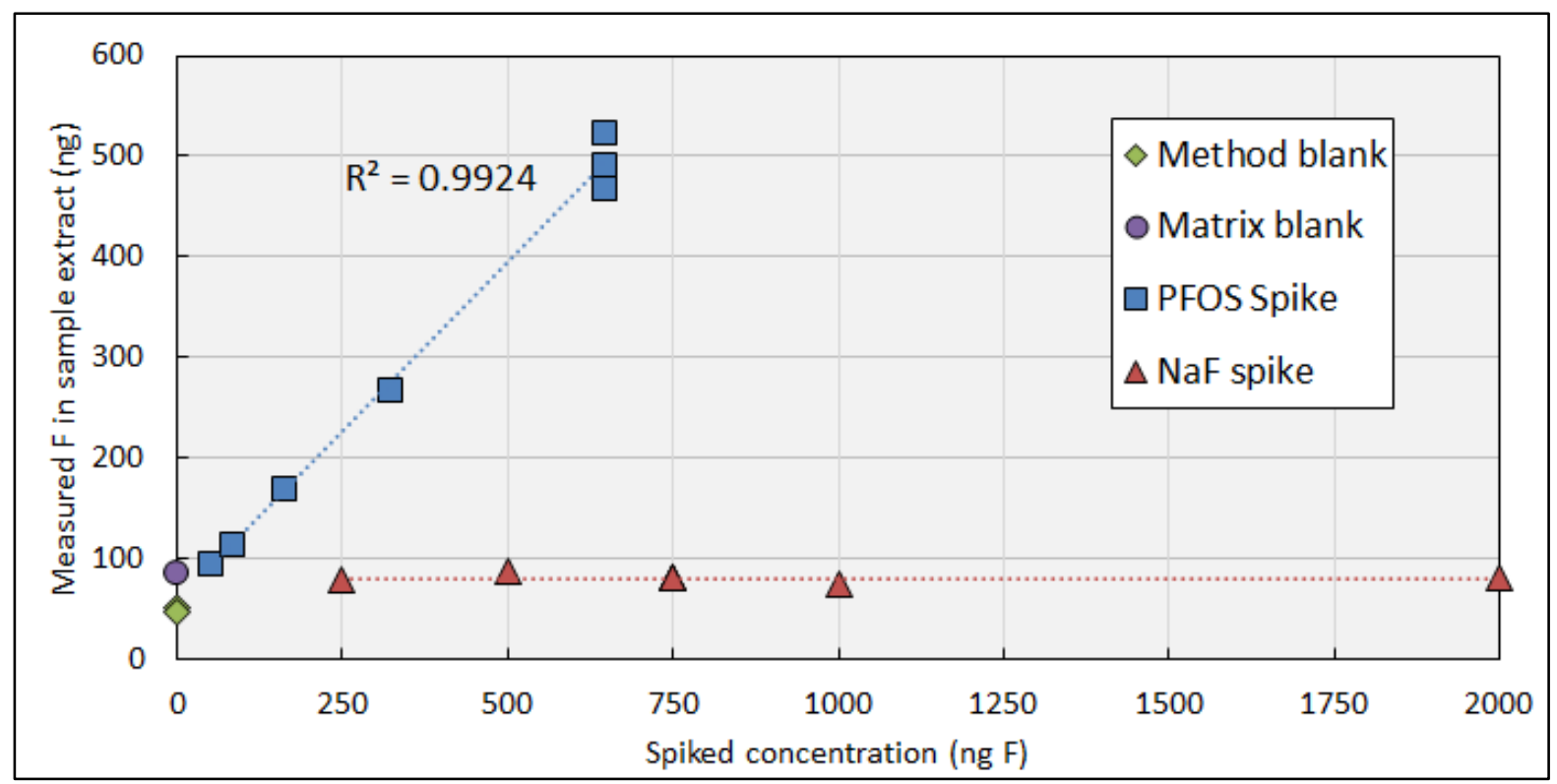

Figure S3. Results from spike/recovery experiments for CIC analysis. Comparison between PFOS- and NaF-spiked samples. 


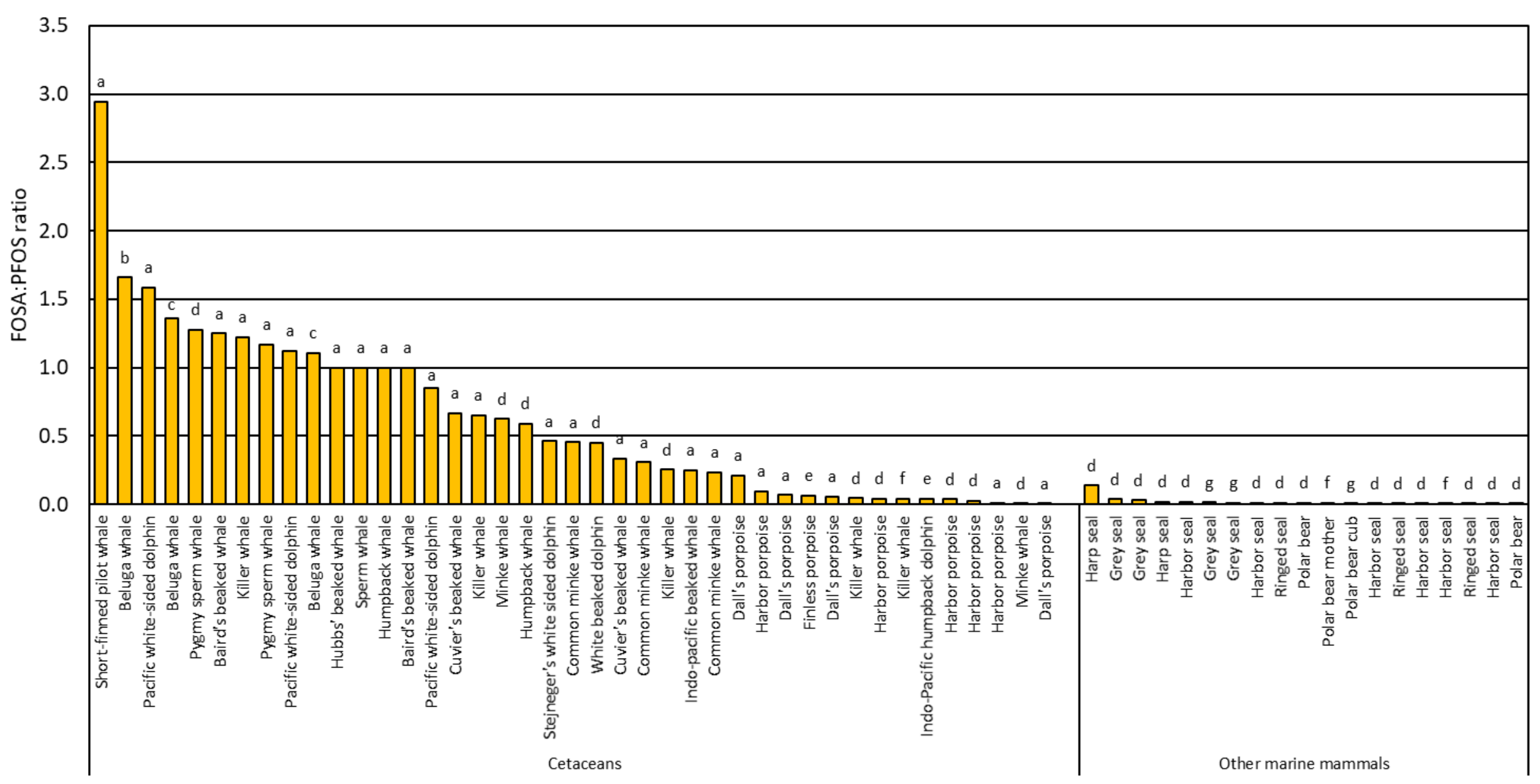

Figure S4. Overview on the FOSA:PFOS ratio for cetaceans and other marine mammals from literature as well as from the present study. ${ }^{\mathrm{a}}$ Fujii et al. ${ }^{6},{ }^{\mathrm{b}}$ Tomy et al. ${ }^{7},{ }^{\mathrm{c}}$ Kelly et al. ${ }^{8},{ }^{\mathrm{d}}$ Present study, ${ }^{\mathrm{e}}$ Yeung et al. ${ }^{9},{ }^{\mathrm{f}}$ Gebbink et al..${ }^{10}$, ${ }^{\mathrm{g}}$ Shaw et al..${ }^{11}$ 


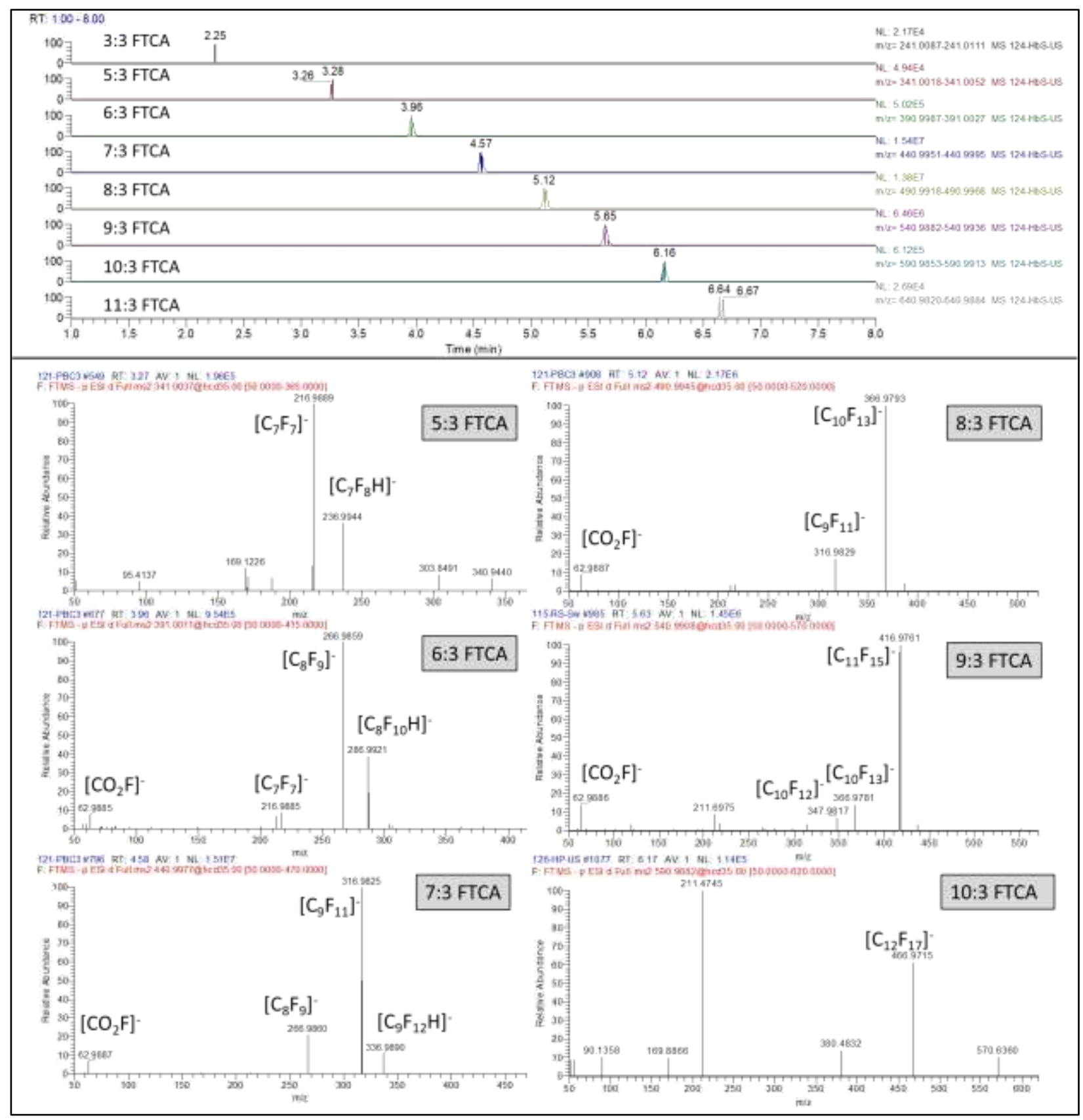

Figure S5. Above the EICs for x:3 FTCAs (class 3) observed in harbor seal from the US are shown. MS/MS spectra including the molecular formulas belonging to the most common peaks. 


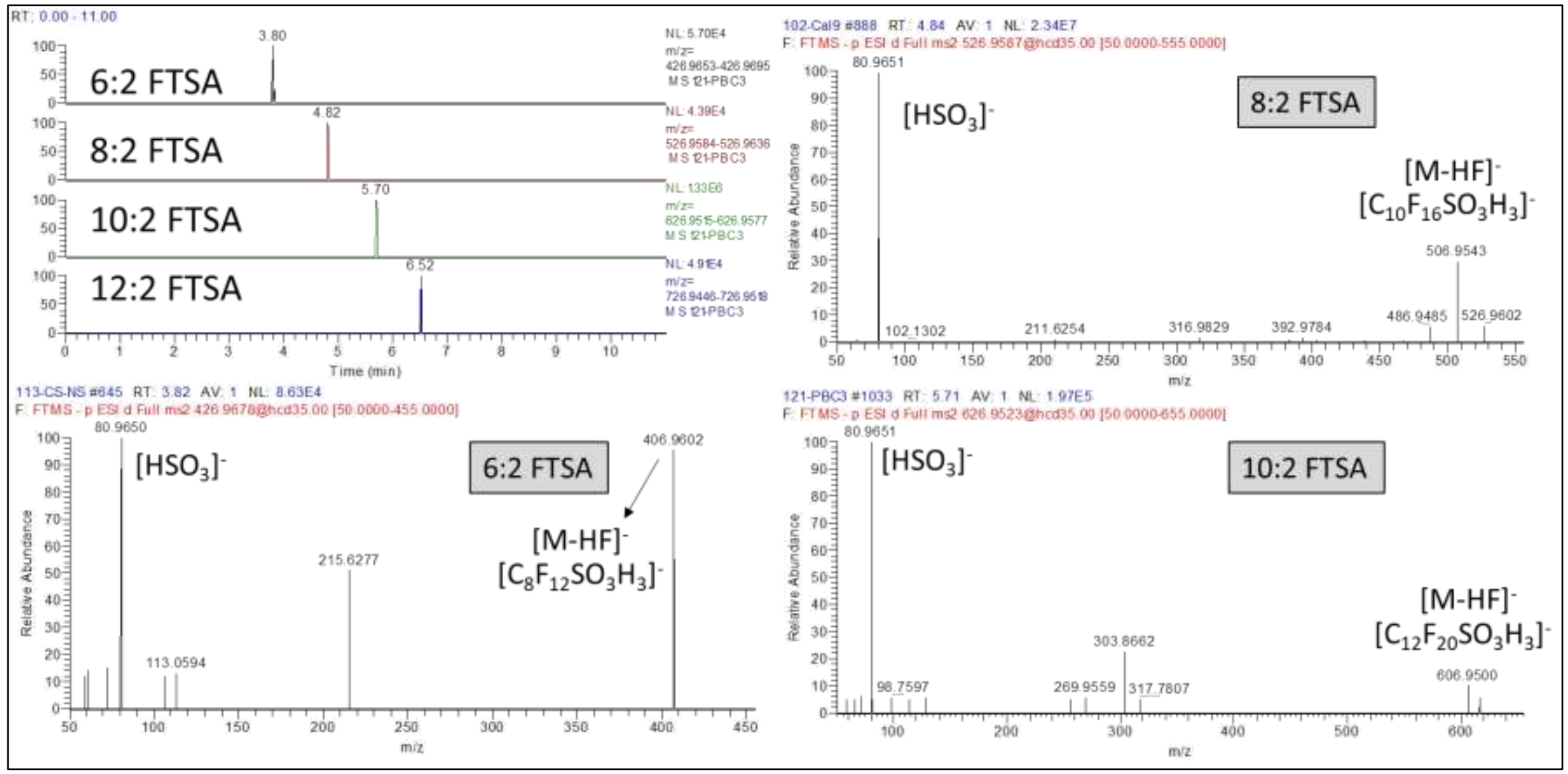

Figure S6. EICs of x:2 FTSAs (class 4) in polar bear cub sample. MS/MS spectra from 6:2 FTSA (grey seal from Sweden), 8:2 FTSA (calibration standard), and 10:2 FTSA (polar bear cub) are shown with molecular formulas assigned to the most common peaks. 


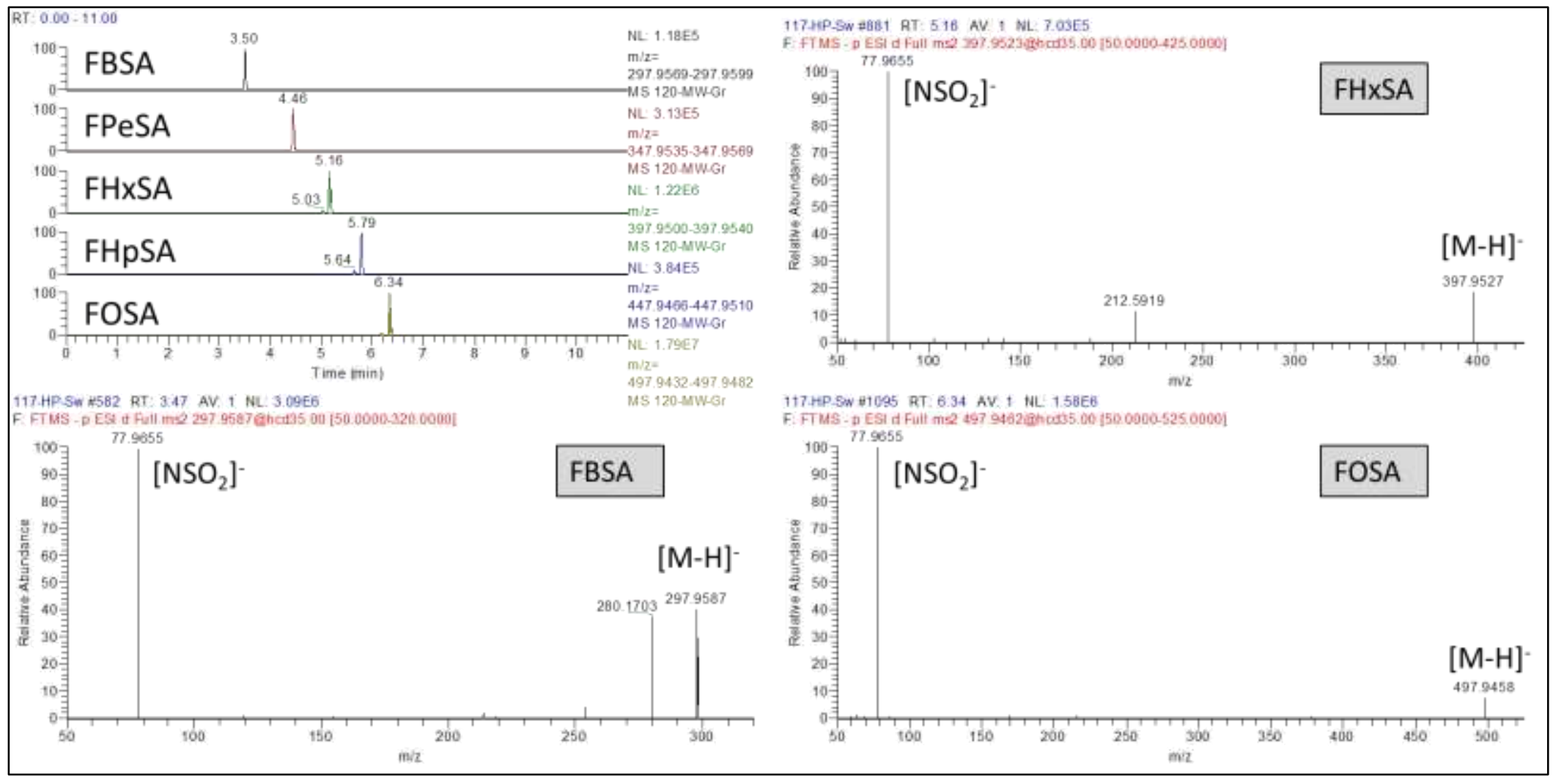

Figure S7. EICs of FASAs (class 5) in minke whale from Greenland. MS/MS spectra for FBSA, FHxSA, and FOSA (harbor porpoise from Sweden) are shown. 


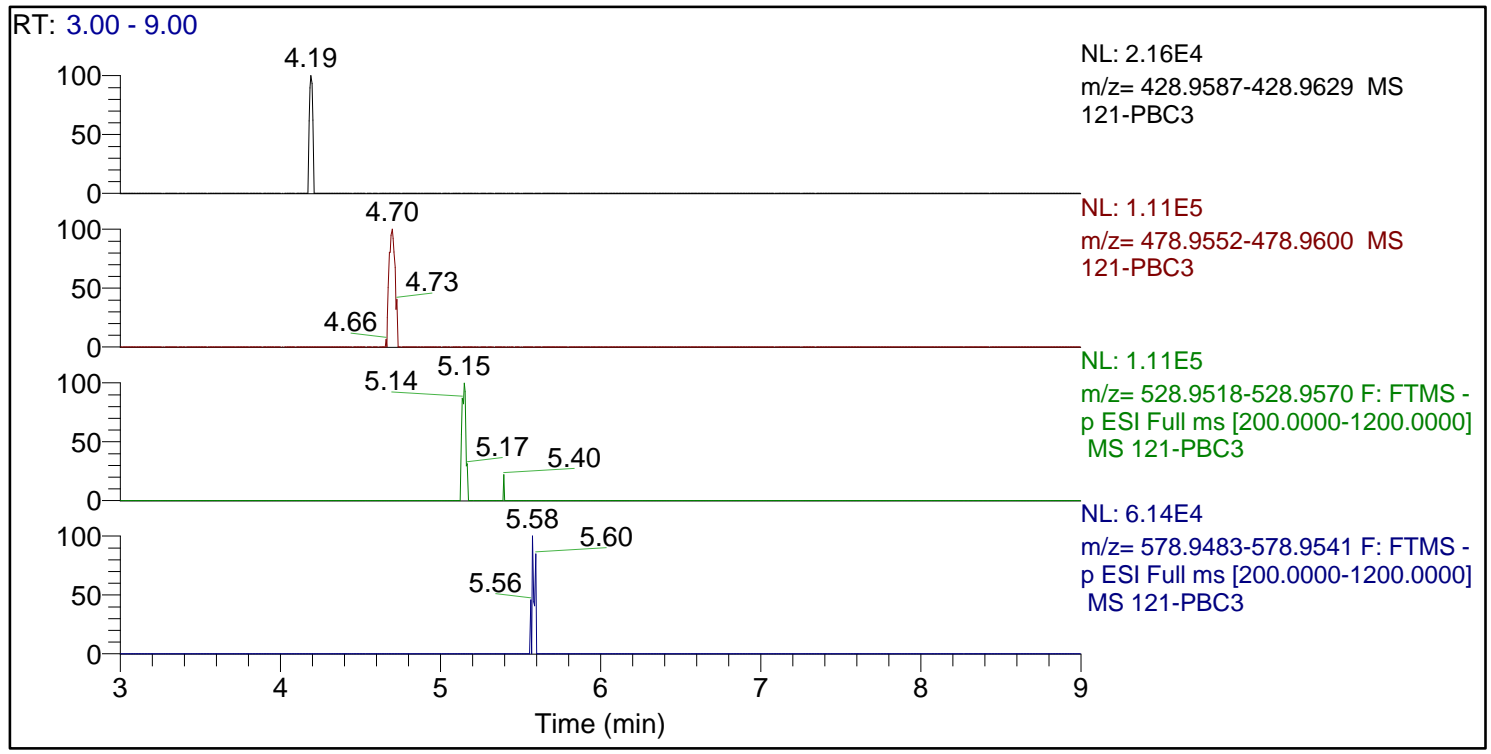

Figure S8. EICs for PFECAs (class 8) in polar bear cub sample. No MS/MS spectra were available. 


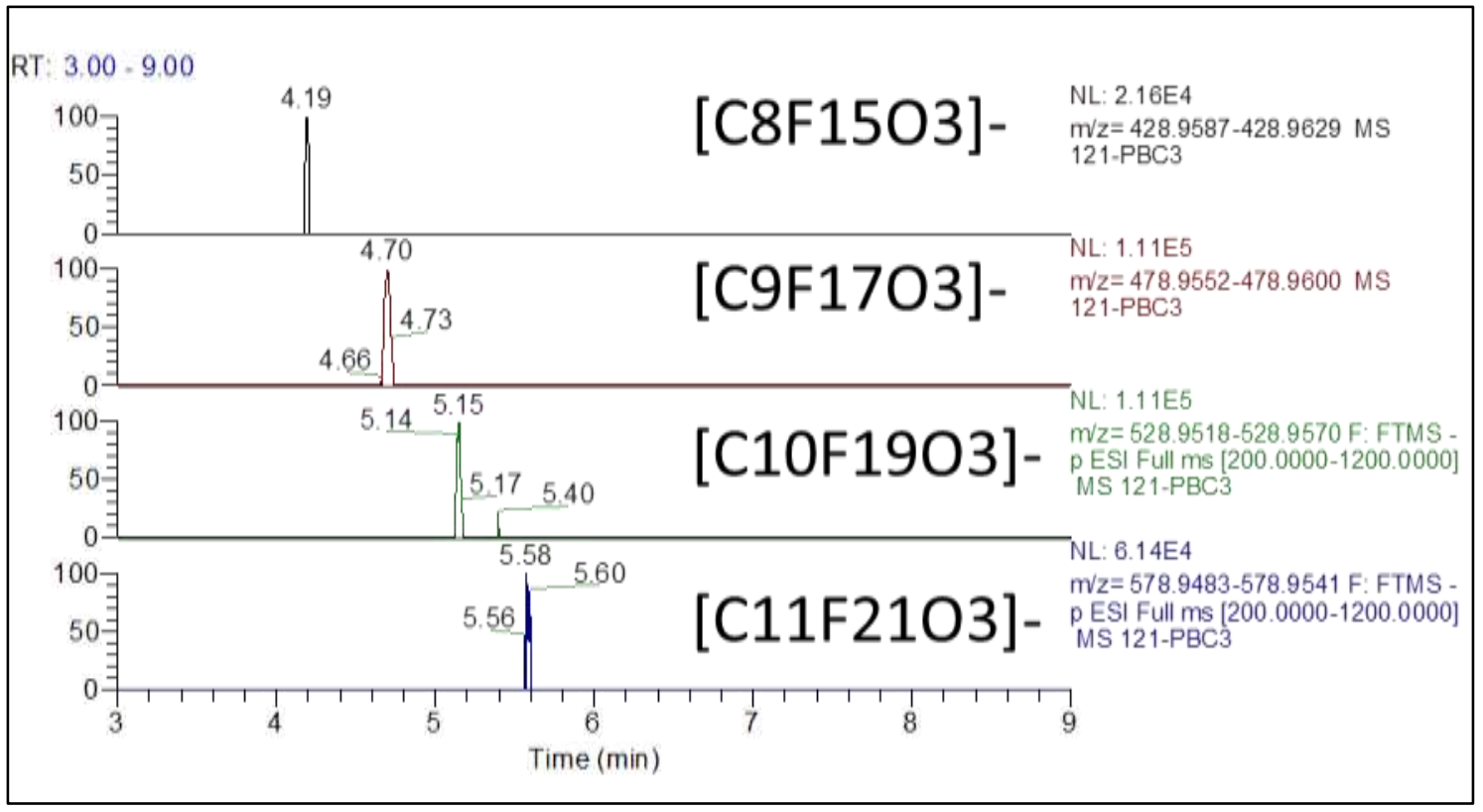

Figure S9. EICs for PFECAs (class 8) in polar bear cub sample. No MS/MS spectra were available. 


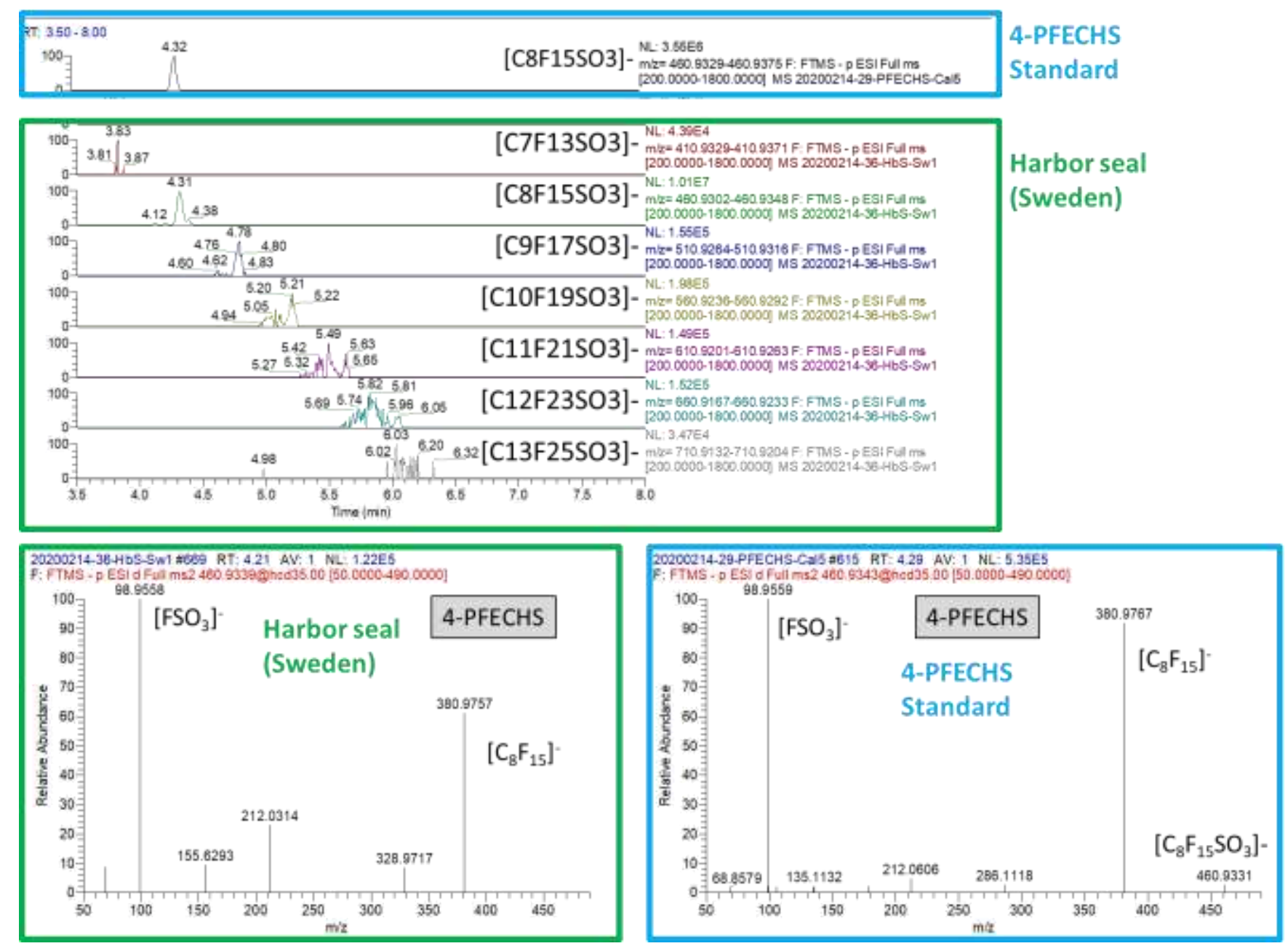

Figure S10. EICs for double-bond/cyclic PFSAs (class 9) in harbor seal sample from Sweden, as well as the MS/MS spectrum for 4PFECHS in harbor seal from Sweden as well as for the purchased 4-PFECHS standard. 


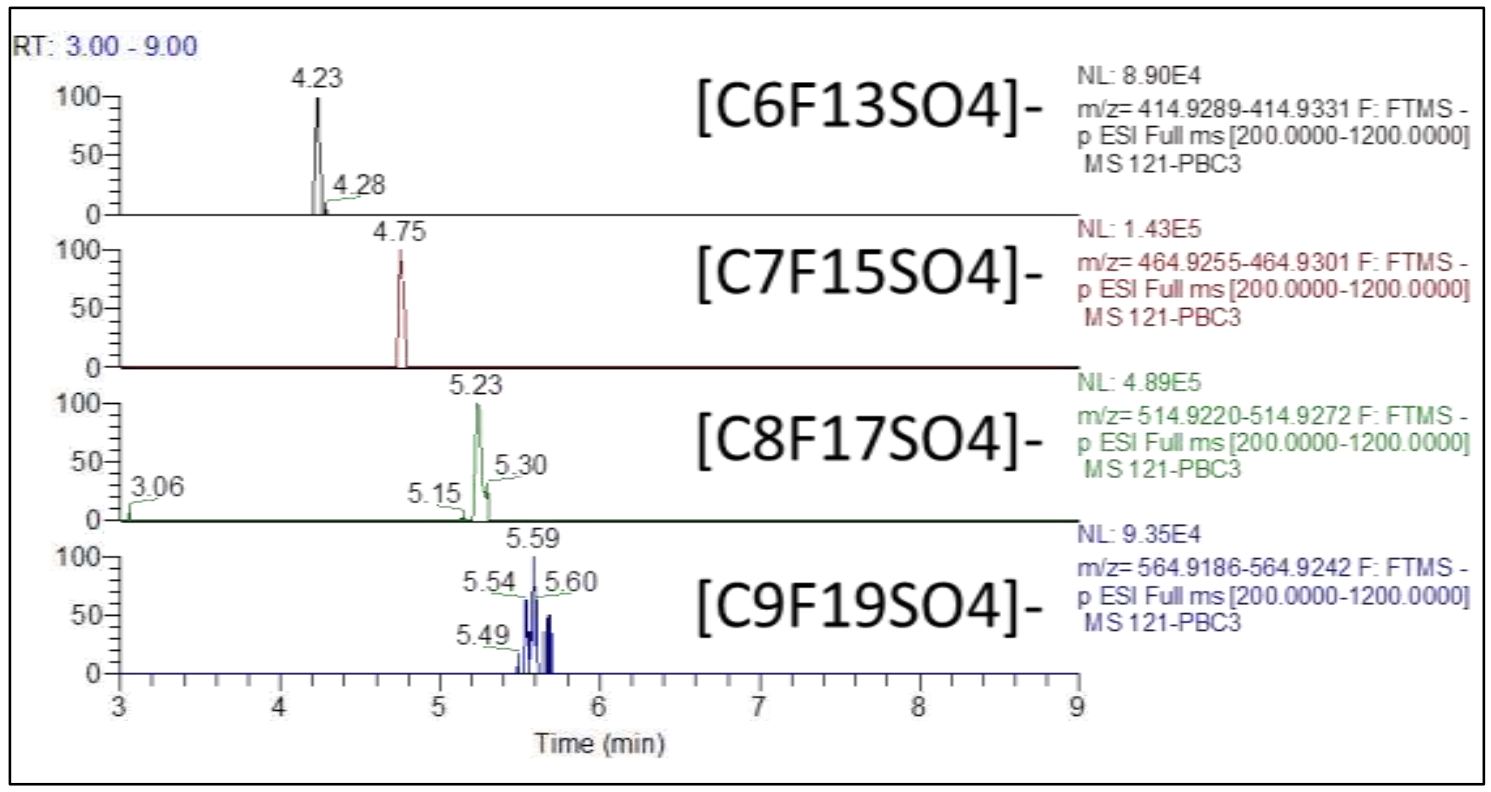

Figure S11. EICs of ether-PFSAs (class 10) in polar bear cub sample. No MS/MS spectra were available. 


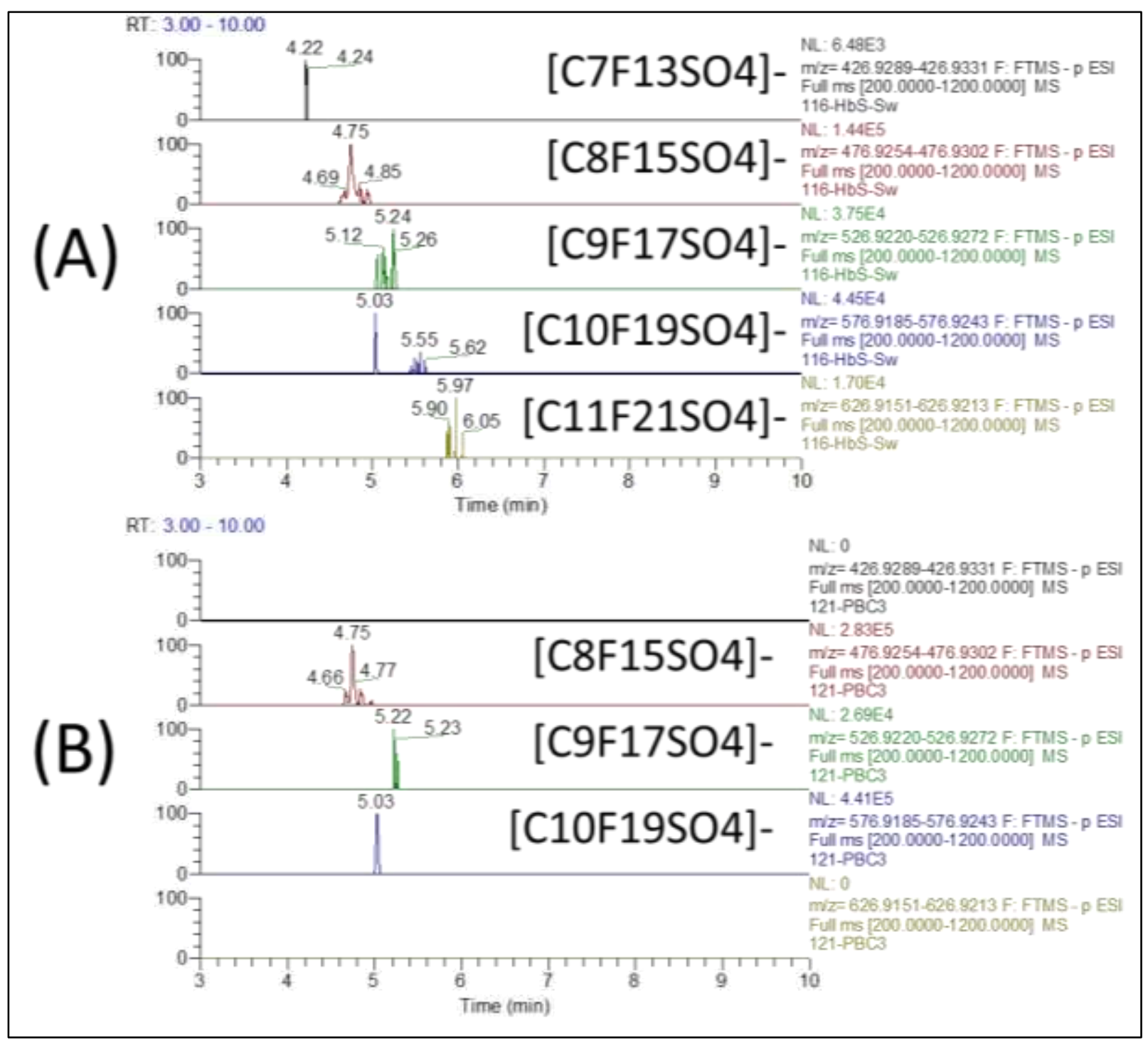

Figure S12. EICs of enol-ether-, cyclic- ether- or carbonyl- PFSAs (class 11) in (A) harbor seal from Sweden and (B) polar bear cub.

No MS/MS spectra were available. 


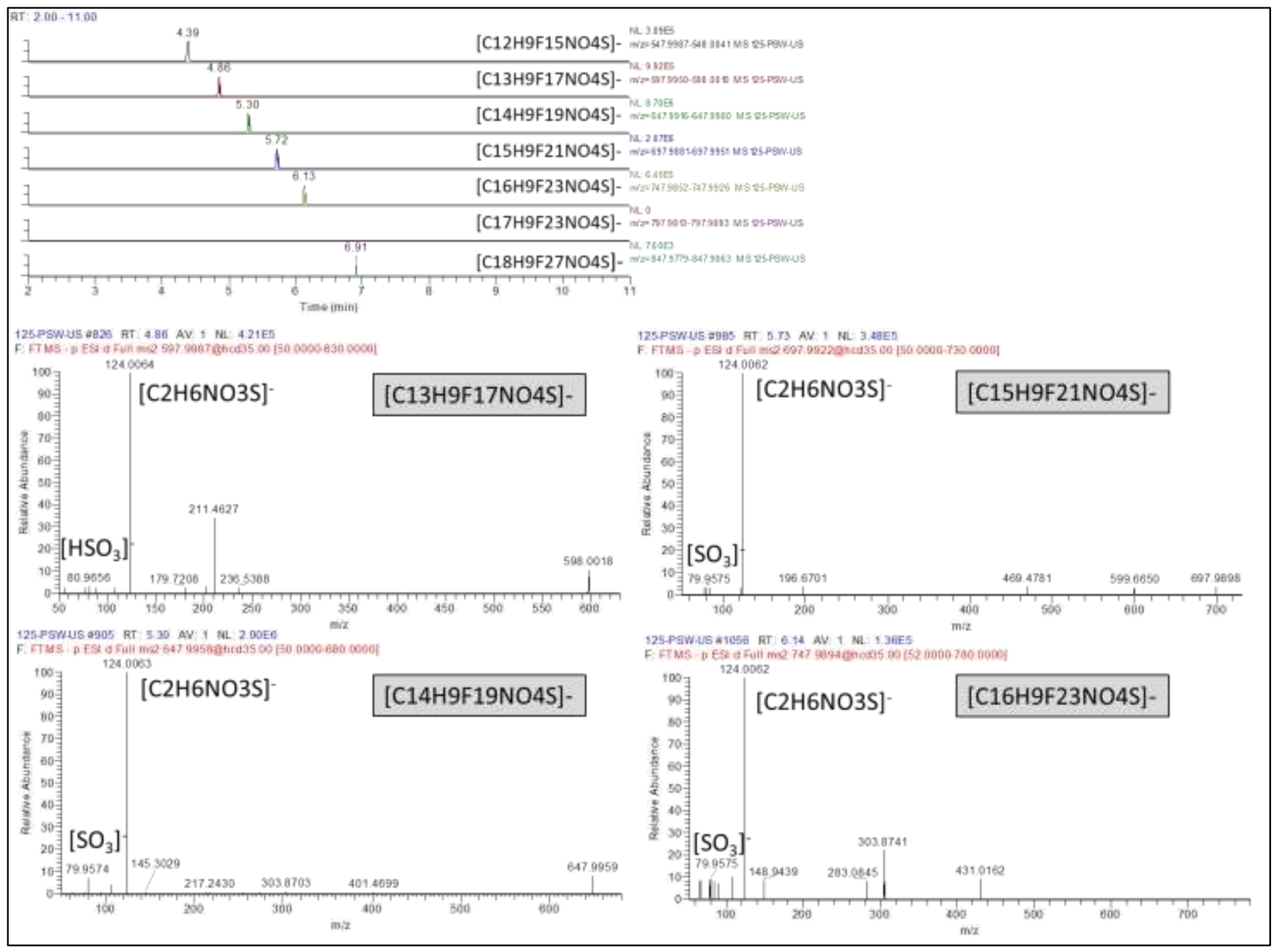

Figure S13. EICs for unknowns (class 12) in pygmy sperm whale. MS/MS spectra for four compounds within class 12 with molecular formulas assigned to the most common fragments. 
Table S1. Detailed overview on the marine mammals that were assessed in this study.

\begin{tabular}{|c|c|c|c|c|c|c|c|c|c|c|}
\hline Sampled by & IDs & Specie & Latin name & Year & Age & $\begin{array}{l}\text { Sex } \\
(M / F)\end{array}$ & Location & $\begin{array}{l}\text { Weight } \\
\text { (kg) }\end{array}$ & $\begin{array}{l}\text { Length } \\
\text { (cm) }\end{array}$ & \\
\hline \multirow{16}{*}{$\begin{array}{c}\text { Susan Shaw } \\
\text { and Michelle } \\
\text { Berger }\end{array}$} & MH 00670 HG, \#7 & Grey seal & Halichoerus grypus & 2000 & Adult & $\mathrm{M}$ & Narragansett Bay, RI, US & 136 & 190 & \multirow{5}{*}{$\begin{array}{l}\frac{0}{0} \\
\frac{0}{0} \\
\frac{0}{8}\end{array}$} \\
\hline & MH 01830 HG,\#17 & Grey seal & Halichoerus grypus & 2001 & Pup & M & Narragansett Bay, RI, US & 22.73 & 99 & \\
\hline & NY 308404 HG, \#245 & Grey seal & Halichoerus grypus & 2004 & Pup & $\mathrm{F}$ & E Long Island, NY, US & 21.7 & 97.2 & \\
\hline & MH 02637 HG, \#314 & Grey seal & Halichoerus grypus & 2002 & Pup & $\mathrm{F}$ & S Massachusetts, US & 18.2 & 96 & \\
\hline & CCSN 02243 HG, \#320 & Grey seal & Halichoerus grypus & 2002 & Subadult & M & Massachusetts, US & 136.4 & 181 & \\
\hline & $\mathrm{MH} 00543 \mathrm{~F}$ PV, 21 & Harbor seal & Phoca vitulina & 2000 & Fetus & $\mathrm{F}$ & Massachusetts Bay, US & 6.4 & 64 & \\
\hline & COA020730PV, \#133 & Harbor seal & Phoca vitulina & 2002 & Adult & $\mathrm{F}$ & Midcoast, ME, US & 54.55 & 140 & \\
\hline & COA060622PV, \#285 & Harbor seal & Phoca vitulina & 2006 & Adult & M & Midcoast, ME, US & 63.6 & 157 & \\
\hline & COA060705PV, \#333 & Harbor seal & Phoca vitulina & 2006 & Pup & $\mathrm{F}$ & E Maine, US & 14.2 & 85 & \\
\hline & COA080717PV-01, \#352 & Harbor seal & Phoca vitulina & 2008 & Yearling & $M$ & Midcoast, ME, US & 16.6 & 100 & \\
\hline & COA060619PP, \#287 & Harbor porpoise & Phocoena phocoena & 2006 & Calf & $\mathrm{F}$ & E Maine, US & 9.9 & 81 & \multirow{5}{*}{$\begin{array}{l}\text { D } \\
\frac{\circ}{0} \\
\frac{0}{0}\end{array}$} \\
\hline & COA060713PP, \#289 & Harbor porpoise & Phocoena phocoena & 2006 & Adult & $\mathrm{F}$ & Midcoast, ME, US & 54.5 & 145 & \\
\hline & COA060905PP, \#340 & Harbor porpoise & Phocoena phocoena & 2006 & Calf & M & E Maine, US & 11.8 & 97 & \\
\hline & COA10100SPP, \#392 & Harbor porpoise & Phocoena phocoena & 2010 & Juvenile & $M$ & E Maine, US & 18.2 & 116.5 & \\
\hline & COA121030PP, \#410 & Harbor porpoise & Phocoena phocoena & 2012 & Juvenile & M & Midcoast, ME, US & 23.3 & 126.8 & \\
\hline & COA071003KB, \#358 & Pygmy sperm whale & Kogia breviceps & 2007 & Calf & $\mathrm{F}$ & Midcoast, ME & 35 & 135 & \\
\hline \multirow{11}{*}{$\begin{array}{l}\text { Anna Roos, } \\
\text { Mads Peter } \\
\text { Heide- } \\
\text { Jørgensen, } \\
\text { Aqqalu } \\
\text { Rosing-Asvid, } \\
\text { Kristin Laidre }\end{array}$} & PAX16/0331 & Harp seal & Pagophilus groenlandicus & 2016 & $\begin{array}{l}\text { Adult } \\
\text { (pregnant) }\end{array}$ & $\mathrm{F}$ & Nuuk (Kobbefjord) (West Greenland) & 93 & 145 & \\
\hline & \multirow{5}{*}{ PAX16/0329 } & Ringed seal & Pusa hispida & 2013 & - & - & Illulisat (North-West Greenland) & - & - & \multirow{5}{*}{$\begin{array}{l}\text { D } \\
\stackrel{0}{0} \\
\frac{0}{D} \\
\stackrel{2}{2}\end{array}$} \\
\hline & & Ringed seal & Pusa hispida & 2013 & - & - & Illulisat (North-West Greenland) & - & - & \\
\hline & & Ringed seal & Pusa hispida & 2013 & - & - & Illulisat (North-West Greenland) & - & - & \\
\hline & & Ringed seal & Pusa hispida & 2013 & - & - & Illulisat (North-West Greenland) & - & - & \\
\hline & & Ringed seal & Pusa hispida & 2013 & - & - & Illulisat (North-West Greenland) & - & - & \\
\hline & \multirow{5}{*}{ PAX16/0327 } & Harbor porpoise & Phocoena phocoena & 2009 & - & - & Maniitsoq, West Greenland & - & - & \multirow{5}{*}{ 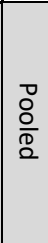 } \\
\hline & & Harbor porpoise & Phocoena phocoena & 2009 & - & - & Maniitsoq, West Greenland & - & - & \\
\hline & & Harbor porpoise & Phocoena phocoena & 2009 & - & - & Maniitsoq, West Greenland & - & - & \\
\hline & & Harbor porpoise & Phocoena phocoena & 2009 & - & - & Maniitsoq, West Greenland & - & - & \\
\hline & & Harbor Porpoise & Phocoena phocoena & 2009 & - & - & Maniitsoq, West Greenland & - & - & \\
\hline
\end{tabular}




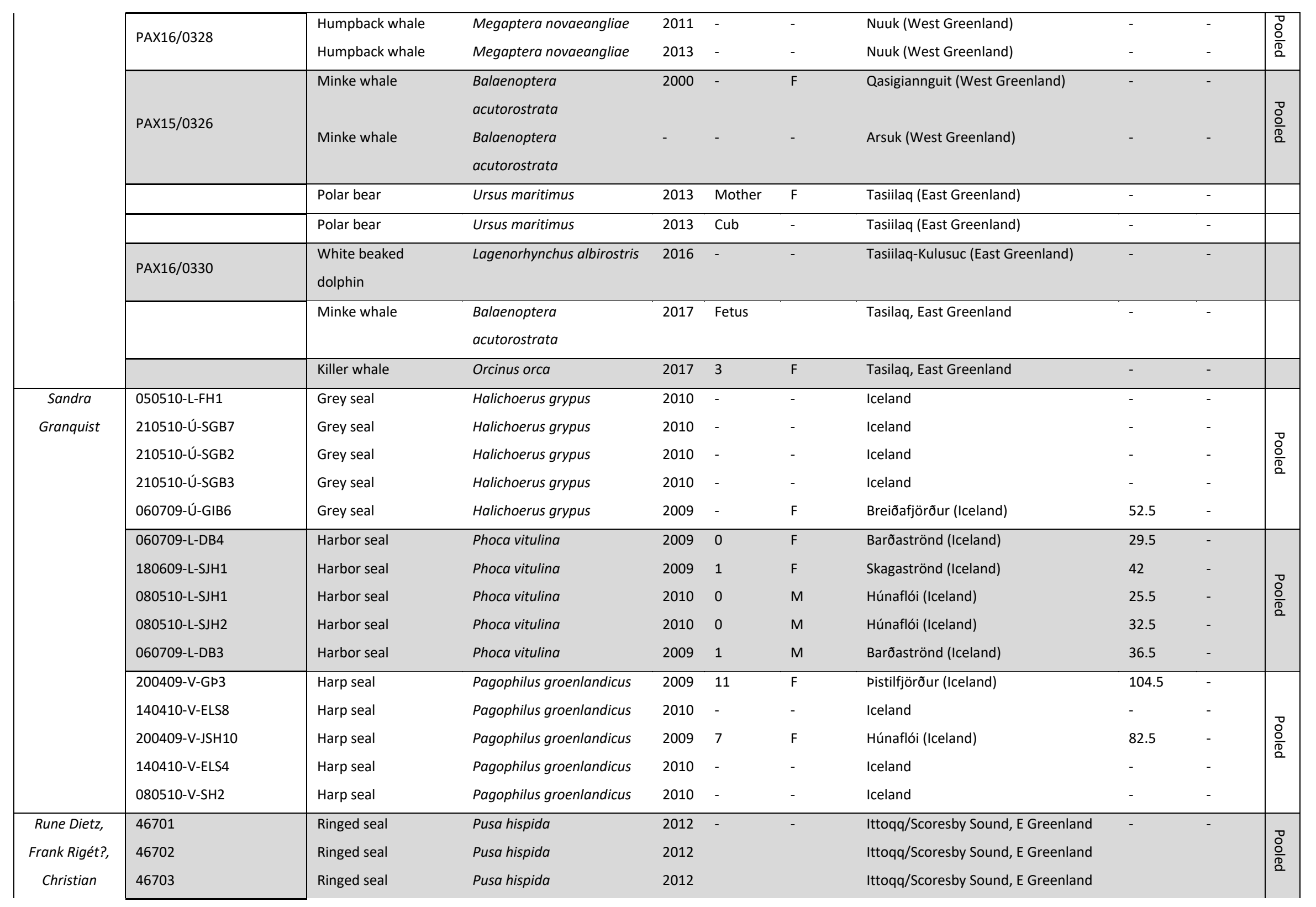




\begin{tabular}{|c|c|c|c|c|c|c|c|c|c|c|}
\hline Sonne and & 46706 & Ringed seal & Pusa hispida & 2012 & & & Ittoqq/Scoresby Sound, E Greenland & & & \\
\hline Aqqalu & 46709 & Ringed seal & Pusa hispida & 2012 & & & Ittoqq/Scoresby Sound, E Greenland & & & \\
\hline \multirow[t]{19}{*}{ Rosing-Asvid } & 46711 & Ringed seal & Pusa hispida & 2012 & & & Ittoqq/Scoresby Sound, E Greenland & & & \\
\hline & 46712 & Ringed seal & Pusa hispida & 2012 & & & Ittoqq/Scoresby Sound, E Greenland & & & \\
\hline & 46714 & Ringed seal & Pusa hispida & 2012 & & & Ittoqq/Scoresby Sound, E Greenland & & & \\
\hline & 46717 & Ringed seal & Pusa hispida & 2012 & & & Ittoqq/Scoresby Sound, E Greenland & & & \\
\hline & 46726 & Ringed seal & Pusa hispida & 2012 & & & Ittoqq/Scoresby Sound, E Greenland & & & \\
\hline & 35143 & Killer whale & Orcinus orca & 2013 & $\mathrm{Ad}$ & $\mathrm{F}$ & Tasilaq/Ammassalik, East Greenland & \multirow[t]{6}{*}{-} & \multirow[t]{6}{*}{-} & \multirow{6}{*}{ 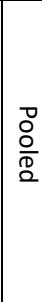 } \\
\hline & 35144 & Killer whale & Orcinus orca & 2013 & SubAd & M & Tasilaq/Ammassalik, East Greenland & & & \\
\hline & 48732 & Killer whale & Orcinus orca & 2013 & $\mathrm{Ad}$ & M & Tasilaq/Ammassalik, East Greenland & & & \\
\hline & 48733 & Killer whale & Orcinus orca & 2013 & $\mathrm{Ad}$ & $\mathrm{F}$ & Tasilaq/Ammassalik, East Greenland & & & \\
\hline & 48734 & Killer whale & Orcinus orca & 2013 & Fetus & M & Tasilaq/Ammassalik, East Greenland & & & \\
\hline & 48735 & Killer whale & Orcinus orca & 2013 & SubAd & $\mathrm{F}$ & Tasilaq/Ammassalik, East Greenland & & & \\
\hline & 46752 & Polar bear & Ursus maritimus & 2012 & 10 & M & Ittoqq/Scoresby Sound, E Greenland & - & - & \multirow{8}{*}{$\begin{array}{l}\frac{0}{2} \\
\frac{\circ}{\circ} \\
\stackrel{0}{\circ}\end{array}$} \\
\hline & 46753 & Polar bear & Ursus maritimus & 2012 & 6 & M & Ittoqq/Scoresby Sound, E Greenland & & & \\
\hline & 46754 & Polar bear & Ursus maritimus & 2201 & 7 & $\mathrm{~F}$ & Ittoqq/Scoresby Sound, E Greenland & & & \\
\hline & 46755 & Polar bear & Ursus maritimus & 2012 & 5 & $\mathrm{~F}$ & Ittoqq/Scoresby Sound, E Greenland & & & \\
\hline & 46756 & Polar bear & Ursus maritimus & 2012 & 2 & $\mathrm{~F}$ & Ittoqq/Scoresby Sound, E Greenland & & & \\
\hline & 46758 & Polar bear & Ursus maritimus & 2012 & 5 & M & Ittoqq/Scoresby Sound, E Greenland & & & \\
\hline & 46759 & Polar bear & Ursus maritimus & 2012 & 5 & M & Ittoqq/Scoresby Sound, E Greenland & & & \\
\hline & 46760 & Polar bear & Ursus maritimus & 2012 & 3 & M & Ittoqq/Scoresby Sound, E Greenland & & & \\
\hline \multirow[t]{10}{*}{ Anna Roos } & A2012/05463 & Grey seal & Halichoerus grypus & 2012 & Yearling & $\mathrm{M}$ & Sweden & 117 & 34.2 & \multirow{5}{*}{ 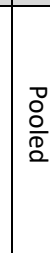 } \\
\hline & A2013/05230 & Grey seal & Halichoerus grypus & 2013 & Yearling & M & Sweden & 113 & 34 & \\
\hline & A2015/05571 & Grey seal & Halichoerus grypus & 2015 & 21 & M & Sweden & 145 & - & \\
\hline & A2015/05614 & Grey seal & Halichoerus grypus & 2015 & 12 & $\mathrm{~F}$ & Sweden & 115 & - & \\
\hline & A2016/05270 & Grey seal & Halichoerus grypus & 2016 & 1 & M & Sweden & 50.4 & - & \\
\hline & A2015/05387 & Harbor seal & Phoca vitulina & 2015 & Yearling & $\mathrm{F}$ & Sweden & 18.9 & 94 & \multirow{5}{*}{ 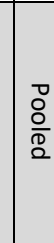 } \\
\hline & A2015/05390 & Harbor seal & Phoca vitulina & 2015 & Yearling & M & Sweden & 19.7 & 97 & \\
\hline & A2016/05109 & Harbor seal & Phoca vitulina & 2015 & Adult & M & Sweden & 105 & - & \\
\hline & A2016/05167 & Harbor seal & Phoca vitulina & 2015 & Adult & $F$ & Sweden & 75 & - & \\
\hline & A2016/05316 & Harbor seal & Phoca vitulina & 2015 & Yearling & $\mathrm{F}$ & Sweden & 75 & - & \\
\hline
\end{tabular}




\begin{tabular}{|c|c|c|c|c|c|c|c|c|c|}
\hline A2014/05650 & Ringed seal & Phoca hispida & 2014 & 23 & $\mathrm{~F}$ & Northern Baltic & - & - & \\
\hline A2015/05591 & Ringed seal & Phoca hispida & 2015 & Adult & M & Northern Baltic & - & - & \multirow{4}{*}{$\begin{array}{l}0 \\
\frac{8}{\mathbb{D}} \\
\stackrel{0}{2}\end{array}$} \\
\hline A2016/05110 & Ringed seal & Phoca hispida & 2015 & Yearling & $\mathrm{M}$ & Northern Baltic & 31.8 & 100.5 & \\
\hline A2016/05126 & Ringed seal & Phoca hispida & 2015 & Adult & $\mathrm{F}$ & Northern Baltic & 41.6 & 132 & \\
\hline A2016/05133 & Ringed seal & Phoca hispida & 2015 & Yearling & $\mathrm{F}$ & Northern Baltic & 37.1 & 108 & \\
\hline A2015/05283 & Harbor porpoise & Phocoena phocoena & 2011 & Adult & $\mathrm{M}$ & Southern Baltic & - & 145 & \multirow{5}{*}{$\frac{8}{8}$} \\
\hline A2016/05526 & Harbor porpoise & Phocoena phocoena & 2016 & Subadult & M & Southern Baltic & - & 124 & \\
\hline A2016/05528 & Harbor porpoise & Phocoena phocoena & - & Juvenile & $\mathrm{F}$ & Southern Baltic & - & 114 & \\
\hline A2016/05637 & Harbor porpoise & Phocoena phocoena & 2016 & Adult & M & Southern Baltic & 43 & 141.5 & \\
\hline C2012/00009 & Harbor porpoise & Phocoena phocoena & 2011 & Adult & M & Southern Baltic & 45 & 154.5 & \\
\hline $\begin{array}{l}\text { A2016/05633 } \\
\text { liver } 3439\end{array}$ & Grey seal & Halichoerus grypus & 2016 & (pregnant) & $\mathrm{F}$ & Sweden - Gävleborgs län & - & - & \\
\hline
\end{tabular}


Table S2. Convention on International Trade in Endangered Species of Wild Fauna and Flora (CITES) numbers for export permissions.

\begin{tabular}{|c|c|c|}
\hline CITES nr & Species & Name \\
\hline 17GL1167082 & Polar bear & Anna Roos \\
\hline 17GL1167083 & Harbor porpoise & Anna Roos \\
\hline 17GL1167084 & Humpback whale & Anna Roos \\
\hline 17GL1167085 & Minke whale & Anna Roos \\
\hline 17GL1167088 & Killer whale & Anna Roos \\
\hline 17GL1167090 & Humpback whale & Anna Roos \\
\hline 17GL1167098 & White beaked dolphins, East Greenland & Anna Roos \\
\hline 17US18692C/9 & Pygmy sperm whale & Susan Shaw and Michelle Berger \\
\hline 17US18692C/9 & Harbor porpoise & Susan Shaw and Michelle Berger \\
\hline
\end{tabular}


Table S3. Target compounds according to their compound class, acronyms, and molecular formula.

\begin{tabular}{|c|c|c|c|}
\hline & Name & Acronym & Molecular formula \\
\hline \multirow{14}{*}{ 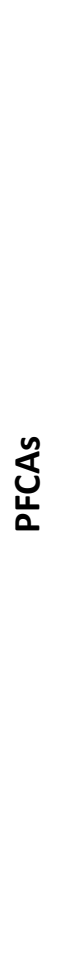 } & Perfluorobutanoic acid & PFBA & $\mathrm{C}_{4} \mathrm{~F}_{7} \mathrm{O}_{2} \mathrm{H}$ \\
\hline & Perfluoropentanoic acid & PFPeA & $\mathrm{C}_{5} \mathrm{~F}_{9} \mathrm{O}_{2} \mathrm{H}$ \\
\hline & Perfluorohexanoaic acid & PFHXA & $\mathrm{C}_{6} \mathrm{~F}_{11} \mathrm{O}_{2} \mathrm{H}$ \\
\hline & Perfluoroheptanoic acid & PFHpA & $\mathrm{C}_{7} \mathrm{~F}_{13} \mathrm{O}_{2} \mathrm{H}$ \\
\hline & Perfluorooctanoaic acid & $\mathrm{PFOA}^{\mathrm{L}+\mathrm{Br}}$ & $\mathrm{C}_{8} \mathrm{~F}_{15} \mathrm{O}_{2} \mathrm{H}$ \\
\hline & Perfluorononanoaic acid & PFNA & $\mathrm{C}_{9} \mathrm{~F}_{17} \mathrm{O}_{2} \mathrm{H}$ \\
\hline & Perfluorodecanoaic acid & PFDA & $\mathrm{C}_{10} \mathrm{~F}_{19} \mathrm{O}_{2} \mathrm{H}$ \\
\hline & Perfluoroundecanoic acid & PFUnDA & $\mathrm{C}_{11} \mathrm{~F}_{21} \mathrm{O}_{2} \mathrm{H}$ \\
\hline & Perfluorododecanoic acid & PFDoDA & $\mathrm{C}_{12} \mathrm{~F}_{23} \mathrm{O}_{2} \mathrm{H}$ \\
\hline & Perfluorotridecanoic acid & PFTrDA & $\mathrm{C}_{13} \mathrm{~F}_{25} \mathrm{O}_{2} \mathrm{H}$ \\
\hline & Perfluorotetradecanoic acid & PFTeDA & $\mathrm{C}_{14} \mathrm{~F}_{27} \mathrm{O}_{2} \mathrm{H}$ \\
\hline & Perfluoropentadecanoic acid & PFPeDA & $\mathrm{C}_{15} \mathrm{~F}_{29} \mathrm{O}_{2} \mathrm{H}$ \\
\hline & Perfluorohexadecanoic acid & PFHxDA & $\mathrm{C}_{16} \mathrm{~F}_{31} \mathrm{O}_{2} \mathrm{H}$ \\
\hline & Perfluorooctadecanoic acid & PFOcDA & $\mathrm{C}_{18} \mathrm{~F}_{35} \mathrm{O}_{2} \mathrm{H}$ \\
\hline \multirow{7}{*}{$\frac{n}{\frac{4}{4}}$} & Perfluorobutane sulfonic acid & PFBS & $\mathrm{C}_{4} \mathrm{~F}_{9} \mathrm{SO}_{3} \mathrm{H}$ \\
\hline & Perfluoropentane sulfonic acid & PFPeS & $\mathrm{C}_{5} \mathrm{~F}_{11} \mathrm{SO}_{3} \mathrm{H}$ \\
\hline & Perfluorohexane sulfonic acid & $\mathrm{PFHxS} \mathrm{S}^{\mathrm{L}+\mathrm{Br}}$ & $\mathrm{C}_{6} \mathrm{~F}_{13} \mathrm{SO}_{3} \mathrm{H}$ \\
\hline & Perfluoroheptane sulfonic acid & PFHpS & $\mathrm{C}_{7} \mathrm{~F}_{15} \mathrm{SO}_{3} \mathrm{H}$ \\
\hline & Perfluorooctane sulfonic acid & $\mathrm{PFOS}^{\mathrm{L}+\mathrm{Br}}$ & $\mathrm{C}_{8} \mathrm{~F}_{17} \mathrm{SO}_{3} \mathrm{H}$ \\
\hline & Perfluorononane sulfonic acid & PFNS & $\mathrm{C}_{9} \mathrm{~F}_{19} \mathrm{SO}_{3} \mathrm{H}$ \\
\hline & Perfluorodecane sulfonic acid & $\mathrm{PFDS}^{\mathrm{L}+\mathrm{Br}}$ & $\mathrm{C}_{10} \mathrm{~F}_{21} \mathrm{SO}_{3} \mathrm{H}$ \\
\hline
\end{tabular}




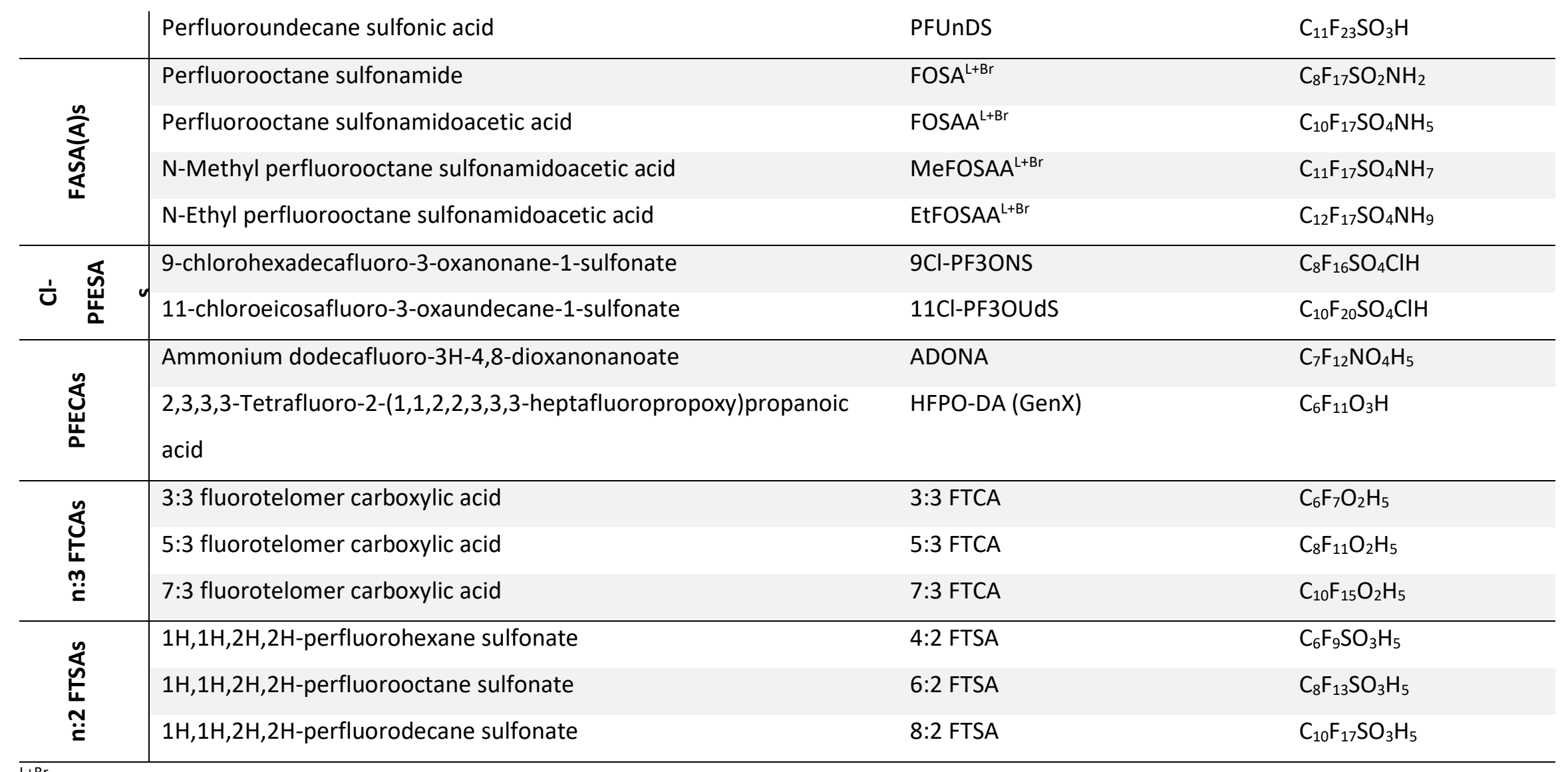

$\overline{\mathrm{L}+\mathrm{Br}}=$ both linear and branched isomers are analyzed. 
Table S4. Target analytes with their quantification and qualifications ions as well as the internal standard used for quantification. L indicates linear ions, and br indicates branched ions. All ISs were purchased from Wellington Laboratories (Guelph, Canada).

\begin{tabular}{|c|c|c|c|c|c|c|c|c|}
\hline Target Analyte & $\begin{array}{c}\text { Average RT } \\
\text { (min) }\end{array}$ & $\begin{array}{c}\text { Precursor } \\
\text { ion }\end{array}$ & $\begin{array}{l}\text { Quantitative } \\
\text { product ion }\end{array}$ & $\begin{array}{l}\text { Qualitative } \\
\text { product ion }\end{array}$ & IS & IS transition & $\begin{array}{l}\text { Quantification } \\
\text { standard }\end{array}$ & $\begin{array}{c}\text { Data } \\
\text { quality }^{1}\end{array}$ \\
\hline PFBA & 0.66 & 213 & 169 & 149 & ${ }^{13} \mathrm{C}_{4}-\mathrm{PFBA}$ & $217 / 172$ & PFBA & $Q$ \\
\hline PFPeA & 1.46 & 263 & 219 & 169 & ${ }^{13} \mathrm{C}_{2}-\mathrm{PFHxA}$ & $266 / 222$ & PFPeA & Q \\
\hline PFHXA & 2.19 & 313 & 269 & 119 & ${ }^{13} \mathrm{C}_{2}-\mathrm{PFH} \times \mathrm{A}$ & $315 / 270$ & PFHXA & Q \\
\hline PFHpA & 2.63 & 363 & 319 & 169 & ${ }^{13} \mathrm{C}_{4}$-PFOA & $367 / 322$ & PFHpA & semiQ \\
\hline L-PFOA & 2.98 & 413 & 369 & 169 & ${ }^{13} \mathrm{C}_{4}$-PFOA & $417 / 372$ & PFOA & $Q$ \\
\hline br-PFOA & 2.92 & 413 & 369 & 169 & ${ }^{13} \mathrm{C}_{4}$-PFOA & $417 / 372$ & PFOA & semiQ \\
\hline PFNA & 3.30 & 463 & 419 & 219 & ${ }^{13} C_{5}$-PFNA & $468 / 423$ & PFNA & Q \\
\hline PFDA & 3.58 & 513 & 469 & 269 & ${ }^{13} \mathrm{C}_{2}$-PFDA & $515 / 470$ & PFDA & Q \\
\hline PFUnDA & 3.87 & 563 & 519 & 269 & ${ }^{13} \mathrm{C}_{2}$-PFUnDA & $565 / 520$ & PFUnDA & $Q$ \\
\hline PFDoDA & 4.14 & 613 & 569 & 169 & ${ }^{13} \mathrm{C}_{2}$-PFDoDA & $615 / 570$ & PFDoDA & $Q$ \\
\hline PFTrDA & 4.40 & 662.9 & 619 & 169 & ${ }^{13} \mathrm{C}_{2}$-PFDoDA & $615 / 570$ & PFTrDA & semiQ \\
\hline PFTeDA & 4.65 & 712.9 & 669 & 169 & ${ }^{13} C_{2}$-PFDoDA & $615 / 570$ & PFTeDA & semiQ \\
\hline PFPeDA & 4.90 & 762.9 & 719 & 169 & ${ }^{13} \mathrm{C}_{2}$-PFDoDA & $615 / 570$ & PFHxDA & semiQ \\
\hline PFHxDA & 5.15 & 813 & 769 & 169 & ${ }^{13} \mathrm{C}_{2}$-PFDoDA & $615 / 570$ & PFHxDA & semiQ \\
\hline PFOcDA & 5.59 & 913 & 869 & 169 & ${ }^{13} \mathrm{C}_{2}$-PFDoDA & $615 / 570$ & PFOcDA & semiQ \\
\hline PFBS & 2.12 & 298.9 & 80 & 99 & ${ }^{18} \mathrm{O}_{2}-\mathrm{PFHxS}$ & $403 / 84$ & PFBS & $Q$ \\
\hline PFPeS-80 & $2.57^{2}$ & 348.9 & 80 & 99 & ${ }^{18} \mathrm{O}_{2}-\mathrm{PFHxS}$ & $403 / 84$ & PFHXS & semiQ \\
\hline PFPeS-99 & $2.57^{2}$ & 348.9 & 99 & 80 & ${ }^{18} \mathrm{O}_{2}$-PFHxS & $403 / 84$ & PFHXS & semiQ \\
\hline L-PFHxS & 3.02 & 398.9 & 80 & 99 & ${ }^{18} \mathrm{O}_{2}-\mathrm{PFHxS}$ & $403 / 84$ & PFHXS & $Q$ \\
\hline
\end{tabular}




\begin{tabular}{|c|c|c|c|c|c|c|c|c|}
\hline br-PFHxS & 2.97 & 399 & 80 & 99 & ${ }^{18} \mathrm{O}_{2}-\mathrm{PFHxS}$ & $403 / 84$ & PFHxS & semiQ \\
\hline PFHpS-80 & 3.36 & 448.9 & 80 & 99 & ${ }^{18} \mathrm{O}_{2}$-PFHxS & $403 / 84$ & PFHxS & semiQ \\
\hline PFHpS-99 & 3.36 & 448.9 & 99 & 80 & ${ }^{18} \mathrm{O}_{2}$-PFHxS & $403 / 84$ & PFHxS & semiQ \\
\hline L-PFOS-80 & 3.66 & 498.9 & 80 & 99 & ${ }^{13} \mathrm{C}_{4}$-PFOS & $503 / 80$ & PFOS & $Q$ \\
\hline br-PFOS-80 & 3.57 & 498.9 & 80 & 99 & ${ }^{13} \mathrm{C}_{4}$-PFOS & $503 / 80$ & PFOS & semiQ \\
\hline L-PFOS-99 & 3.66 & 498.9 & 99 & 80 & ${ }^{13} \mathrm{C}_{4}$-PFOS & $503 / 80$ & PFOS & $Q$ \\
\hline br-PFOS-99 & 3.57 & 498.9 & 99 & 80 & ${ }^{13} \mathrm{C}_{4}$-PFOS & $503 / 80$ & PFOS & semiQ \\
\hline PFNS-80 & 3.83 & 548.9 & 80 & 99 & ${ }^{13} \mathrm{C}_{4}$-PFOS & $503 / 80$ & PFOS & semiQ \\
\hline PFNS-99 & 3.83 & 548.9 & 99 & 80 & ${ }^{13} \mathrm{C}_{4}$-PFOS & $503 / 80$ & PFOS & semiQ \\
\hline L-PFDS & 4.23 & 598.9 & 80 & 99 & ${ }^{13} \mathrm{C}_{4}$-PFOS & $503 / 80$ & PFDS & $Q$ \\
\hline br-PFDS & 4.17 & 598.9 & 80 & 99 & ${ }^{13} \mathrm{C}_{4}$-PFOS & $503 / 80$ & PFDS & semiQ \\
\hline PFUnDS-80 & $4.63^{2}$ & 648.9 & 80 & 99 & ${ }^{13} \mathrm{C}_{4}$-PFOS & $503 / 80$ & PFDS & semiQ \\
\hline PFUnDS-99 & $4.63^{2}$ & 648.9 & 99 & 80 & ${ }^{13} \mathrm{C}_{4}$-PFOS & $503 / 80$ & PFDS & semiQ \\
\hline L-FOSA & 4.40 & 497.9 & 78 & 169 & ${ }^{13} \mathrm{C}_{8}$-FOSA & $506 / 78$ & FOSA & $Q$ \\
\hline br-FOSA & 4.34 & 497.9 & 78 & 169 & ${ }^{13} \mathrm{C}_{8}$-FOSA & $506 / 78$ & FOSA & semiQ \\
\hline L-FOSAA & 3.49 & 555.9 & 498 & 419 & $\mathrm{D}_{3}-\mathrm{MeFOSAA}$ & $573 / 419$ & FOSAA & $Q$ \\
\hline br-FOSAA & 3.44 & 555.9 & 498 & 419 & $\mathrm{D}_{3}-\mathrm{MeFOSAA}$ & $573 / 419$ & FOSAA & semiQ \\
\hline L-MeFOSAA & 3.62 & 570 & 419 & 483 & $\mathrm{D}_{3}-\mathrm{MeFOSAA}$ & $573 / 419$ & MeFOSAA & $\mathrm{Q}$ \\
\hline br-MeFOSAA & 3.56 & 570 & 419 & 483 & $\mathrm{D}_{3}-\mathrm{MeFOSAA}$ & $573 / 419$ & MeFOSAA & semiQ \\
\hline L-EtFOSAA & 3.74 & 584 & 419 & 526 & $\mathrm{D}_{5}$-EtFOSAA & $589 / 419$ & EtFOSAA & Q \\
\hline br-EtFOSAA & 3.69 & 584 & 419 & 526 & $\mathrm{D}_{5}$-EtFOSAA & $589 / 419$ & EtFOSAA & semiQ \\
\hline 9Cl-PF3ONS & 3.86 & 531 & 351 & 83 & ${ }^{13} \mathrm{C}_{4}$-PFOS & $503 / 80$ & 9Cl-PF3ONS & semiQ \\
\hline 11Cl-PF3OUdS & 4.42 & 631 & 451 & 83 & ${ }^{13} \mathrm{C}_{4}$-PFOS & $503 / 80$ & 11Cl-PF3OUdS & semiQ \\
\hline
\end{tabular}




\begin{tabular}{|c|c|c|c|c|c|c|c|c|}
\hline ADONA & 2.75 & 377 & 251 & 85 & ${ }^{13} \mathrm{C}_{4}$-PFOS & $503 / 80$ & ADONA & semiQ \\
\hline HFPO-DA & 2.36 & 329 & 169 & 185 & ${ }^{13} \mathrm{C}_{4}$-PFOA & $417 / 372$ & HFPO-DA & semiQ \\
\hline 3:3 FTCA & 0.95 & 241 & 117 & 177 & ${ }^{13} \mathrm{C}_{4}$-PFOA & $417 / 372$ & 3:3 FTCA & semiQ \\
\hline 5:3 FTCA & 2.50 & 341 & 237 & 217 & ${ }^{13} \mathrm{C}_{4}$-PFOA & $417 / 372$ & 5:3 FTCA & semiQ \\
\hline 7:3 FTCA & 3.30 & 441 & 337 & 148 & ${ }^{13} \mathrm{C}_{4}$-PFOA & $417 / 372$ & 7:3 FTCA & semiQ \\
\hline 4:2 FTSA & 2.03 & 327 & 307 & 80.6 & ${ }^{13} C_{2}-6: 2$ FTSA & $429 / 409$ & $4: 2$ FTSA & semiQ \\
\hline 6:2 FTSA & 2.86 & 427 & 407 & 80.6 & ${ }^{13} C_{2}-6: 2$ FTSA & $429 / 409$ & $6: 2$ FTSA & $Q$ \\
\hline 8:2 FTSA & 3.46 & 527 & 507 & 80.6 & ${ }^{13} C_{2}-6: 2$ FTSA & $429 / 409$ & $8: 2$ FTSA & semiQ \\
\hline${ }^{13} \mathrm{C}_{8}-$ PFOA $^{3}$ & 2.98 & 421 & 376 & & & & & \\
\hline${ }^{13} \mathrm{C}_{8}-\mathrm{PFOS}^{3}$ & 3.66 & 507 & 80 & & & & & \\
\hline
\end{tabular}

${ }^{1} \mathrm{Q}=$ quantitative, semiQ = semi-quantitative for compounds lacking authentic standards and/or analogous ISs.

${ }^{2}$ Estimated retention time, based on retention times of adjacent PFSAs.

${ }^{3{ }^{13}} \mathrm{C}_{8}$-PFOA and ${ }^{13} \mathrm{C}_{8}$-PFOS were used as recovery internal standards. 
Table S5. Mobile phase gradient program for targeted analysis. Flow rate was $0.4 \mathrm{ml} / \mathrm{min}$, column temperature $50^{\circ} \mathrm{C}$, injection volume $5 \mu \mathrm{l}$.

\begin{tabular}{|c|c|c|}
\hline Time (min) & Mobile phase $A^{1}(\%)$ & Mobile phase $B^{2}(\%)$ \\
\hline 0.0 & 90 & 10 \\
\hline 0.5 & 90 & 10 \\
\hline 5.0 & 20 & 80 \\
\hline 5.1 & 0 & 100 \\
\hline 8.0 & 0 & 100 \\
\hline 10.0 & 90 & 10 \\
\hline
\end{tabular}

${ }^{1}$ Mobile phase A: $90 \%$ water and $10 \%$ acetonitrile containing $2 \mathrm{mM}$ ammonium acetate. ${ }^{2}$ Mobile phase B: $99 \%$ acetonitrile and $1 \%$ water containing 2 mM ammonium acetate. 
Table S6. Limit of quantification (LOQ) for all compounds determined by the lowest calibration concentration.

\begin{tabular}{|c|c|c|c|c|c|}
\hline Compound & LOQ (ng/g) & Compound & LOQ (ng/g) & Compound & LOQ (ng/g) \\
\hline PFBA & 0.814 & PFOcDA & 15.1 & L-FOSAA $^{a}$ & 1.16 \\
\hline PFPeA & 0.290 & $\mathrm{PFBS}^{\mathrm{a}}$ & 2.37 & L-MeFOSAA & 0.826 \\
\hline PFHXA & 0.290 & L-PFHxS & 0.014 & L-Et-FOSAA & 0.296 \\
\hline PFHpA & 0.290 & br-PFHxS & 0.014 & 9Cl-PF3ONS & 0.840 \\
\hline L-PFOA & 0.290 & $\mathrm{PFHpS}^{\mathrm{b}}$ & 0.014 & $11 \mathrm{Cl}-\mathrm{PF} 30 \mathrm{OUS}$ & 0.042 \\
\hline PFNA & 0.290 & L-PFOS- $80^{a}$ & 5.81 & ADONA & 0.816 \\
\hline PFDA & 0.042 & br-PFOS- $80^{a}$ & 5.81 & HFPO-DA & 40.6 \\
\hline PFUnDA & 0.290 & L-PFOS-99a & 6.28 & 3:3 FTCA & 105 \\
\hline PFDoDA & 0.290 & br-PFOS-99a & 6.28 & 5:3 FTCA & 106 \\
\hline PFTrDA & 0.290 & L-PFDS & 0.040 & 7:3 FTCA & 5.61 \\
\hline PFTeDA & 0.814 & br-PFDS ${ }^{b}$ & 0.040 & $4: 2$ FTSA & 0.820 \\
\hline PFPeDA $^{b}$ & 0.290 & L-FOSA & 0.302 & 6:2 FTSA & 0.826 \\
\hline PFHxDA & 0.814 & br-FOSA ${ }^{b}$ & 0.302 & $8: 2$ FTSA & 40.9 \\
\hline
\end{tabular}

${ }^{a}$ Compounds that were present in the method blanks and for these the LOQ was determined alternatively by calculating the average contamination concentration plus ten times the standard deviation. ${ }^{\mathrm{b}}$ Compounds that were not present in the calibration curve, but that were present in the samples. 
Table S7. Comparison of NIST serum standard reference material (SRM) 1957, reported reference values, and results from method used in the present study.

\begin{tabular}{|c|c|c|c|c|}
\hline Compound & $\begin{array}{l}\text { NIST certificate values } \\
\qquad(\mathrm{ng} / \mathrm{g})\end{array}$ & Gebbink et al. ${ }^{12}(\mathrm{ng} / \mathrm{g})$ & Yeung et al. ${ }^{13}$ (ng/g) & Present study (ng/g) \\
\hline PFHpA & $0.305 \pm 0.036$ & $0.2 \pm 0.02$ & $0.2 \pm 0.1$ & $<0.29$ \\
\hline PFOA & $5 \pm 0.4$ & $3.86 \pm 0.13$ & $4.1 \pm 0.3$ & $5.0 \pm 0.1$ \\
\hline PFNA & $0.88 \pm 0.068$ & $0.72 \pm 0.04$ & $0.8 \pm 0.1$ & $0.8 \pm 0.2$ \\
\hline PFDA & $0.39 \pm 0.1$ & $0.24 \pm 0.01$ & $0.3 \pm 0.0$ & $0.3 \pm 0.1$ \\
\hline PFUnDA & $0.174 \pm 0.031$ & $0.11 \pm 0.01$ & $0.1 \pm 0.1$ & $<0.29$ \\
\hline PFDoDA & - & $0.017 \pm 0.003$ & - & - \\
\hline PFTrDA & - & $0.009 \pm 0.004$ & - & - \\
\hline PFHxS & $4 \pm 0.75$ & $3.25 \pm 0.06$ & $4.1 \pm 0.5$ & $4.0 \pm 0.2$ \\
\hline PFOS & $21.1 \pm 1.2$ & $18.5 \pm 0.7$ & $19.3 \pm 1.2$ & $18.4 \pm 0.5$ \\
\hline FOSA & - & $0.029 \pm 0.007$ & - & - \\
\hline
\end{tabular}

“_" = not detected 
Table S8. Eluent program for the ion chromatography part of the CIC analysis.

\begin{tabular}{l|l} 
Time (min) & Concentration $\mathrm{OH}^{-}(\mathbf{m M})$ \\
\hline
\end{tabular}

\begin{tabular}{c|c}
\hline 0.0 & 8.0 \\
4.0 & 8.0 \\
9.9 & 45.0 \\
10.0 & 100.0 \\
14.0 & 100.0 \\
14.1 & 8.0 \\
20.0 & 8.0 \\
\hline
\end{tabular}


Table S9. Set-up parameters for the HRMS Orbitrap.

Scan parameters

\begin{tabular}{l|l}
\hline Scan typ & Full MS \\
Scan range & $200-1200 \mathrm{~m} / \mathrm{z}$ \\
Fragmentation & None or NCE(35) $(\mathrm{z}=1)$ \\
Resolution & 120000 \\
Polarity & Negative \\
Maximum inject time & $30 / 250$ \\
& \\
\hline
\end{tabular}

\section{HESI source}

Sheath gas flow rate

Aux gas flow rate

Sweep gas flow rate

Spray voltage $(\mathrm{kV})$

Capillary temp. $\left({ }^{\circ} \mathrm{C}\right)$

S-lens RF level

Aux gas heater temp. $\left({ }^{\circ} \mathrm{C}\right)$

30
10
0
3.70
350
55.0
350


Table S10. Concentration (ng/g or ng F/g) of PFAS, EOF and TF for sample from East Greenland and Sweden.

\begin{tabular}{|c|c|c|c|c|c|c|c|c|c|c|c|c|c|}
\hline \multirow[b]{2}{*}{ (ng/g) } & \multicolumn{8}{|c|}{ East Greenland } & \multicolumn{5}{|c|}{ Sweden } \\
\hline & 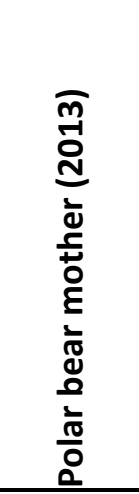 & 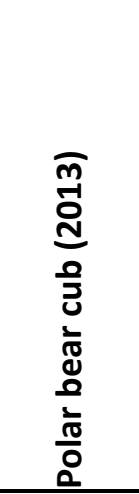 & 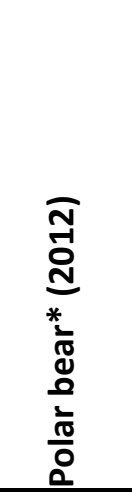 & 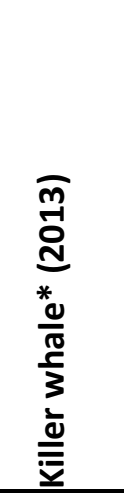 & 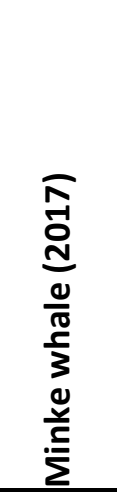 & 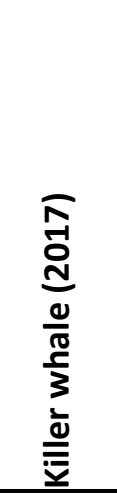 & 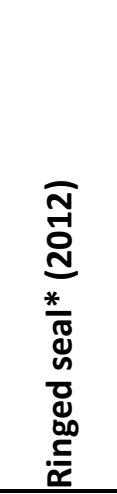 & 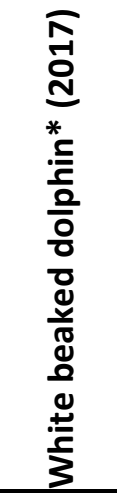 & 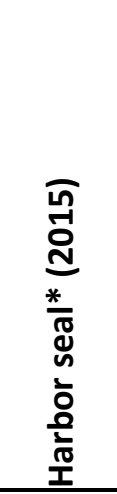 & 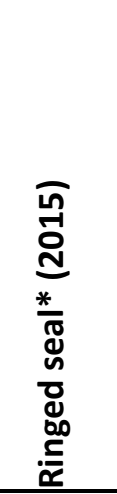 & 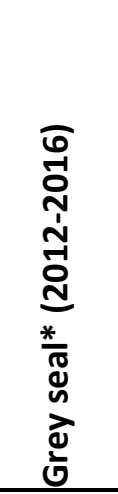 & 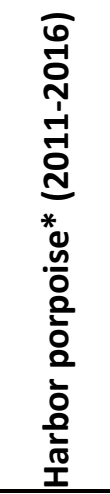 & 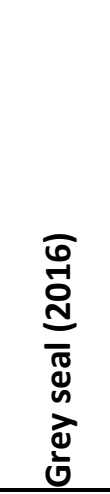 \\
\hline PFHpA & 1.5 & 1.4 & 1.2 & 0.5 & $<0.29$ & $<0.29$ & $<0.29$ & $<0.29$ & $<0.29$ & $<0.29$ & $<0.29$ & $<0.29$ & $<0.29$ \\
\hline PFOA (L) & 55.3 & 53.8 & 27.4 & 0.7 & $<0.29$ & $<0.29$ & 0.8 & 0.5 & 1.8 & 10.6 & 2.1 & $<0.29$ & 0.7 \\
\hline PFNA & 451.1 & 492.9 & 333.9 & 20.2 & 9.0 & 4.5 & 13.7 & 7.7 & 24.7 & 124.8 & 50.5 & 3.2 & 35.9 \\
\hline PFDA & 138.8 & 153.8 & 116.6 & 43.5 & 21.0 & 10.5 & 10.9 & 4.9 & 14.9 & 48.6 & 16.7 & 7.0 & 9.2 \\
\hline PFUnDA & 252.0 & 235.3 & 157.1 & 168.2 & 96.3 & 48.3 & 24.2 & 19.3 & 21.4 & 45.4 & 19.3 & 18.5 & 7.5 \\
\hline PFDoDA & 25.2 & 22.8 & 16.5 & 28.4 & 11.6 & 5.8 & 2.8 & 1.8 & 4.2 & 5.3 & 2.6 & 2.7 & 0.9 \\
\hline PFTrDA & 68.6 & 63.4 & 63.4 & 148.0 & 30.6 & 15.3 & 10.8 & 8.7 & 9.8 & 11.9 & 8.5 & 10.4 & 2.8 \\
\hline PFTeDA & 9.0 & 8.4 & 5.3 & 27.3 & 6.5 & 3.3 & 1.2 & $<0.81$ & 1.3 & 1.4 & 0.9 & 1.9 & $<0.81$ \\
\hline PFPeDA & 19.7 & 17.3 & 16.2 & 78.6 & 12.9 & 6.5 & 3.2 & 2.7 & 2.1 & 2.5 & 0.9 & 2.0 & 1.2 \\
\hline PFHxDA & $<0.81$ & $<0.81$ & $<0.81$ & 0.9 & 1.1 & $<0.81$ & $<0.81$ & $<0.81$ & $<0.81$ & $<0.81$ & $<0.81$ & $<0.81$ & $<0.81$ \\
\hline PFBS & $<2.4$ & $<2.4$ & $<2.4$ & $<2.4$ & $<2.4$ & $<2.4$ & $<2.4$ & $<2.4$ & $<2.4$ & $<2.4$ & $<2.4$ & $<2.4$ & $<2.4$ \\
\hline PFHxS (L+Br) & 20.7 & 22.3 & 20.7 & 2.1 & 0.9 & 0.5 & 0.7 & 0.9 & 7.6 & 3.8 & 1.2 & 0.7 & 0.5 \\
\hline PFHpS & 14.9 & 16.6 & 19.8 & 0.6 & $<0.01$ & $<0.01$ & 0.3 & $<0.01$ & 2.4 & 1.2 & 0.5 & 0.1 & 0.3 \\
\hline PFOS (L+Br) & 1806.5 & 1784.3 & 1864.0 & 230.3 & 115.0 & 57.5 & 99.7 & 29.5 & 857.9 & 483.4 & 327.8 & 146.9 & 172.1 \\
\hline PFDS (L+Br) & 8.8 & 9.9 & 5.6 & 3.1 & 1.3 & 0.7 & 0.2 & 0.1 & 2.8 & 0.8 & 1.0 & 0.1 & $<0.04$ \\
\hline FOSA (L+Br) & 4.5 & 3.4 & 7.8 & 10.4 & 29.2 & 14.6 & 0.5 & 13.2 & 0.7 & $<0.3$ & 3.7 & 5.4 & 5.2 \\
\hline F-53B & $<0.84$ & $<0.84$ & $<0.84$ & $<0.84$ & $<0.84$ & $<0.84$ & $<0.84$ & $<0.84$ & $<0.84$ & $<0.84$ & $<0.84$ & $<0.84$ & $<0.84$ \\
\hline 5:3 FTCA & $<106$ & $<106$ & $<106$ & $<106$ & $<106$ & $<106$ & $<106$ & $<106$ & $<106$ & $<106$ & $<106$ & $<106$ & $<106$ \\
\hline 7:3 FTCA & 1130.8 & 870.7 & 959.4 & 132.5 & 13.7 & 12.6 & 9.2 & $<5.6$ & 31.1 & 67.1 & 6.3 & 72.5 & $<5.6$ \\
\hline
\end{tabular}




\begin{tabular}{|c|c|c|c|c|c|c|c|c|c|c|c|c|c|}
\hline 6:2 FTSA & $<0.83$ & $<0.83$ & $<0.83$ & $<0.83$ & $<0.83$ & $<0.83$ & $<0.83$ & $<0.83$ & $<0.83$ & $<0.83$ & $<0.83$ & $<0.83$ & $<0.83$ \\
\hline ¿PFCAs (ng/g) & 1021.1 & 1049.0 & 737.7 & 516.3 & 189.2 & 94.3 & 67.8 & 45.6 & 80.1 & 250.4 & 101.7 & 45.7 & 58.2 \\
\hline IPFSAs (ng/g) & 1850.8 & 1833.1 & 1910.2 & 236.1 & 117.3 & 58.7 & 100.9 & 30.5 & 870.7 & 489.2 & 330.5 & 147.8 & 172.9 \\
\hline ¿PFASs (ng/g) & 4007.2 & 3756.1 & 3615.1 & 895.3 & 349.3 & 180.2 & 178.5 & 89.3 & 982.5 & 806.8 & 442.2 & 271.4 & 236.3 \\
\hline 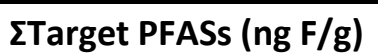 & 2648.8 & 2487.6 & 2379.3 & 613.2 & 238.1 & 122.6 & 119.6 & 60.6 & 640.3 & 535.7 & 291.8 & 178.5 & 156.1 \\
\hline EOF (ng F/g) & 2015.1 & 1818.2 & 3537.5 & 892.8 & 112.8 & 186.1 & 112.7 & $<158$ & 358.8 & 973.2 & 275.1 & $<158$ & 153.4 \\
\hline TF (ng F/g) & 3164.4 & 2992.5 & 5562.9 & 2635.8 & 408.1 & 766.6 & 815.6 & 626.2 & 761.0 & 1085.6 & 684.4 & 743.3 & 568.7 \\
\hline
\end{tabular}


Table S10 continued. Concentration (ng/g or ng F/g) of PFAS, EOF and TF for sample from US Atlantic coast, Iceland and West Greenland.

\begin{tabular}{|c|c|c|c|c|c|c|c|c|c|c|c|c|}
\hline & \multicolumn{4}{|c|}{ US Atlantic coast } & \multicolumn{3}{|c|}{ Iceland } & \multicolumn{5}{|c|}{ West Greenland } \\
\hline & 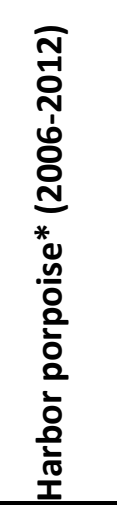 & 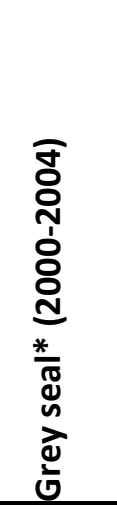 & 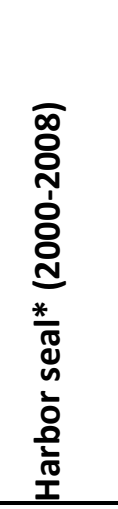 & 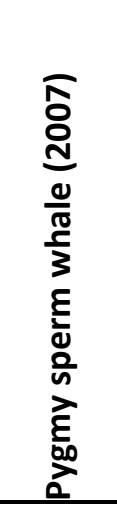 & 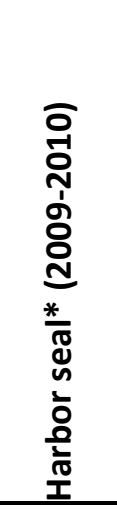 & 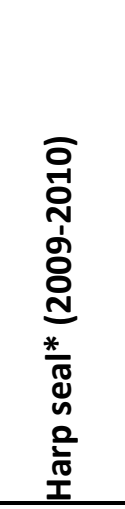 & 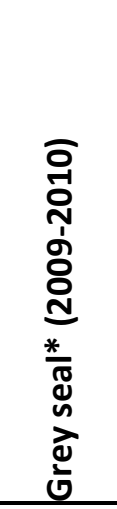 & 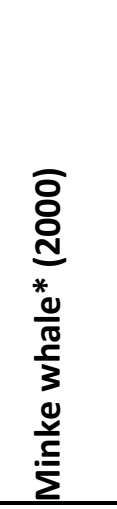 & 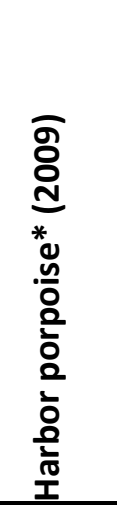 & 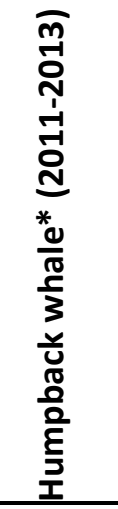 & 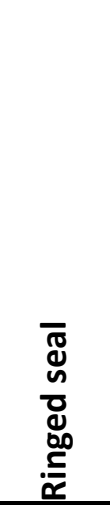 & 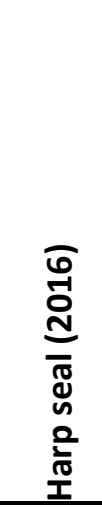 \\
\hline PFHpA & $<0.29$ & $<0.29$ & $<0.29$ & $<0.29$ & $<0.29$ & $<0.29$ & $<0.29$ & $<0.29$ & $<0.29$ & $<0.29$ & $<0.29$ & $<0.29$ \\
\hline PFOA (L) & $<0.29$ & 0.4 & 1.3 & 0.9 & 0.5 & $<0.29$ & $<0.29$ & 0.4 & 0.5 & $<0.29$ & $<0.29$ & 0.4 \\
\hline PFNA & 0.5 & 7.7 & 4.4 & 5.8 & 6.5 & 6.1 & 3.1 & 3.6 & 3.8 & 0.9 & 9.7 & 5.3 \\
\hline PFDA & 5.3 & 8.8 & 2.4 & 1.6 & 4.2 & 4.2 & 1.6 & 3.6 & 7.4 & 5.6 & 5.9 & 4.0 \\
\hline PFUnDA & 7.6 & 21.1 & 5.2 & 7.9 & 19.8 & 11.9 & 5.2 & 14.2 & 28.9 & 28.7 & 18.1 & 10.7 \\
\hline PFDoDA & 1.7 & 4.8 & 1.1 & 1.8 & 2.5 & 1.6 & 1.0 & 1.8 & 3.6 & 3.1 & 2.6 & 1.2 \\
\hline PFTrDA & 6.8 & 15.6 & 7.3 & 18.8 & 10.6 & 7.2 & 4.5 & 6.5 & 14.3 & 12.7 & 10.7 & 4.7 \\
\hline PFTeDA & 1.3 & 2.6 & 1.4 & 4.5 & 1.4 & $<0.81$ & $<0.81$ & $<0.81$ & 1.8 & 2.1 & 1.3 & $<0.81$ \\
\hline PFPeDA & 3.4 & 4.9 & 4.6 & 28.0 & 3.7 & 3.2 & 1.7 & 1.8 & 6.7 & 9.2 & 1.7 & 1.7 \\
\hline PFHxDA & $<0.81$ & $<0.81$ & $<0.81$ & 0.8 & $<0.81$ & $<0.81$ & $<0.81$ & $<0.81$ & $<0.81$ & $<0.81$ & $<0.81$ & $<0.81$ \\
\hline PFBS & $<2.4$ & $<2.4$ & $<2.4$ & $<2.4$ & $<2.4$ & $<2.4$ & $<2.4$ & $<2.4$ & $<2.4$ & $<2.4$ & $<2.4$ & $<2.4$ \\
\hline PFHXS (L+Br) & 0.0 & 0.9 & 1.1 & 0.3 & 0.3 & 0.1 & 0.1 & 0.7 & 0.1 & 0.2 & $<0.01$ & 0.6 \\
\hline PFHpS & $<0.01$ & 0.5 & 0.3 & 0.2 & 0.1 & $<0.01$ & $<0.01$ & 0.1 & 0.1 & $<0.01$ & $<0.01$ & 0.2 \\
\hline PFOS (L+Br) & 69.1 & 168.6 & 50.0 & 6.6 & 37.9 & 21.3 & $<5.8$ & 86.0 & 70.4 & 21.0 & 28.1 & 39.1 \\
\hline PFDS (L+Br) & 0.3 & 8.7 & 0.6 & 0.1 & 0.4 & $<0.04$ & $<0.04$ & 2.6 & 0.2 & 0.6 & 0.2 & 0.1 \\
\hline FOSA (L+Br) & 3.0 & 2.2 & 0.5 & 8.4 & 0.6 & 2.9 & $<0.3$ & 53.5 & 1.7 & 12.3 & $<0.3$ & 0.8 \\
\hline F-53B & $<0.84$ & $<0.84$ & $<0.84$ & $<0.84$ & $<0.84$ & $<0.84$ & $<0.84$ & $<0.84$ & $<0.84$ & $<0.84$ & $<0.84$ & $<0.84$ \\
\hline
\end{tabular}




\begin{tabular}{|c|c|c|c|c|c|c|c|c|c|c|c|c|}
\hline 5:3 FTCA & $<106$ & $<106$ & $<106$ & $<106$ & $<106$ & $<106$ & $<106$ & $<106$ & $<106$ & $<106$ & $<106$ & $<106$ \\
\hline 7:3 FTCA & 189.0 & 38.5 & 193.3 & 20.6 & 10.4 & $<5.6$ & 10.7 & $<5.6$ & $<5.6$ & $<5.6$ & $<5.6$ & $<5.6$ \\
\hline 6:2 FTSA & $<0.83$ & $<0.83$ & $<0.83$ & $<0.83$ & $<0.83$ & $<0.83$ & $<0.83$ & $<0.83$ & $<0.83$ & $<0.83$ & $<0.83$ & $<0.83$ \\
\hline ¿PFCAs (ng/g) & 26.6 & 65.9 & 27.7 & 70.0 & 49.2 & 34.2 & 16.9 & 31.9 & 67.0 & 62.4 & 50.0 & 27.9 \\
\hline ¿PFSAs (ng/g) & 69.5 & 178.6 & 52.0 & 7.1 & 38.6 & 21.4 & 0.1 & 89.4 & 70.7 & 21.8 & 28.3 & 40.0 \\
\hline EPFASs (ng/g) & 288.1 & 285.2 & 273.6 & 106.2 & 99.0 & 58.5 & 27.8 & 174.9 & 139.4 & 96.5 & 78.3 & 68.7 \\
\hline 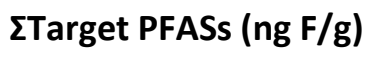 & 188.0 & 188.8 & 178.6 & 73.5 & 67.1 & 40.0 & 19.0 & 115.3 & 94.5 & 66.5 & 53.7 & 46.1 \\
\hline EOF (ng F/g) & 557.8 & 666.3 & 3288.2 & 293.5 & 52.5 & $<71$ & $<71$ & $<158$ & $<158$ & $<158$ & $<158$ & $<158$ \\
\hline TF (ng F/g) & 1560.3 & 1433.7 & 1138.8 & 559.7 & 718.5 & 9196.0 & 298.2 & 1474.5 & 1285.4 & 1487.8 & 539.8 & 811.0 \\
\hline
\end{tabular}




\section{REFERENCES}

(1) Powley, C. R.; George, S. W.; Ryan, T. W.; Buck, R. C. Matrix Effect-Free Analytical Methods for Determination of Perfluorinated Carboxylic Acids in Environmental Matrixes. Anal. Chem. 2005, 77 (19), 6353-6358. https://doi.org/10.1021/ac0508090.

(2) Miyake, Y.; Yamashita, N.; Rostkowski, P.; So, M. K.; Taniyasu, S.; Lam, P. K. S.; Kannan, K. Determination of Trace Levels of Total Fluorine in Water Using Combustion Ion Chromatography for Fluorine: A Mass Balance Approach to Determine Individual Perfluorinated Chemicals in Water. J. Chromatogr. A 2007, 1143 (1-2), 98-104. https://doi.org/10.1016/j.chroma.2006.12.071.

(3) Schultes, L.; Vestergren, R.; Volkova Hellström, K.; Westberg, E.; Jacobson, T.; Benskin, J. P. Per- and Polyfluoroalkyl Substances and Fluorine Mass Balance in Cosmetic Products from the Swedish Market: Implications for Environmental Emissions and Human Exposure. Environ. Sci. Process. Impacts 2018. https://doi.org/10.1039/C8EM00368H.

(4) Schultes, L.; Peaslee, G. F.; Brockman, J. D.; Majumdar, A.; McGuinness, S. R.; Ngwenyama, R. A.; Wilkinson, J. T.; Sandblom, O.; Ngwenyama, R. A.; Benskin, J. P. Total Fluorine Measurements in Food Packaging: How Do Current Methods Perform? Environ. Sci. Technol. Lett. 2019, 6, 73-78. https://doi.org/10.1021/acs.estlett.8b00700.

(5) Miaz, L. T. Development and Application of UHPLC-Orbitrap Method for Quantitative and Suspect Screening of PFASs in Human Serum, Master's thesis, Stockholm University, 2018.

(6) Fujii, Y.; Kato, Y.; Kozai, M.; Matsuishi, T.; Harada, K. H.; Koizumi, A.; Kimura, O.; Endo, T.; Haraguchi, K. Different Profiles of Naturally Produced and Anthropogenic Organohalogens in the Livers of Cetaceans from the Sea of Japan and the North Pacific Ocean. Mar. Pollut. Bull. 2018, $136 \quad$ (August), 230-242. https://doi.org/10.1016/j.marpolbul.2018.08.051.

(7) Tomy, G. T.; Budakowski, W.; Halldorson, T.; Helm, P. A.; Stern, G. A.; Friesen, K.; Pepper, K.; Tittlemier, S. A.; Fisk, A. T. Fluorinated Organic Compounds in an Eastern Arctic Marine Food Web. Environ. Sci. Technol. 2004, 38 (24), 6475-6481. https://doi.org/10.1021/es049620g.

(8) Kelly, B. C.; Ikonomou, M. G.; Blair, J. D.; Surridge, B.; Hoover, D.; Grace, R.; Gobas, F. A. P. C. Perfluoroalkyl Contaminants in an Arctic Marine Food Web: Trophic 
Magnification and Wildlife Exposure. Environ. Sci. Technol. 2009, 43 (11), 4037-4043. https://doi.org/10.1021/es9003894.

(9) Yeung, L. W. Y.; Miyake, Y.; Li, P.; Taniyasu, S.; Kannan, K.; Guruge, K. S.; Lam, P. K. S.; Yamashita, N. Comparison of Total Fluorine, Extractable Organic Fluorine and Perfluorinated Compounds in the Blood of Wild and Pefluorooctanoate (PFOA)-Exposed Rats: Evidence for the Presence of Other Organofluorine Compounds. Anal. Chim. Acta 2009, 635 (1), 108-114. https://doi.org/10.1016/j.aca.2009.01.004.

(10) Gebbink, W. A.; Bossi, R.; Rigét, F. F.; Rosing-Asvid, A.; Sonne, C.; Dietz, R. Observation of Emerging Per- and Polyfluoroalkyl Substances (PFASs) in Greenland Marine Mammals. Chemosphere 2016, 144, 2384-2391. https://doi.org/10.1016/j.chemosphere.2015.10.116.

(11) Shaw, S.; Berger, M. L.; Brenner, D.; Tao, L.; Wu, Q.; Kannan, K. Specific Accumulation of Perfluorochemicals in Harbor Seals (Phoca Vitulina Concolor) from the Northwest Atlantic. $\quad$ Chemosphere 2009, $74 \quad$ (8), 1037-1043. https://doi.org/10.1016/j.chemosphere.2008.10.063.

(12) Gebbink, W. A.; Glynn, A.; Darnerud, P. O.; Berger, U. Perfluoroalkyl Acids and Their Precursors in Swedish Food: The Relative Importance of Direct and Indirect Dietary Exposure. Environ. $\quad$ Pollut. $\quad$ 2015, 198,115 . https://doi.org/10.1016/j.envpol.2014.12.022.

(13) Yeung, L. W. Y.; De Silva, A. O.; Loi, E. I. H.; Marvin, C. H.; Taniyasu, S.; Yamashita, N.; Mabury, S. A.; Muir, D. C. G.; Lam, P. K. S. Perfluoroalkyl Substances and Extractable Organic Fluorine in Surface Sediments and Cores from Lake Ontario. Environ. Int. 2013, 59 (2013), 389-397. https://doi.org/10.1016/j.envint.2013.06.026. 